\title{
On the coverage of the pMSSM by simplified model results
}

\author{
Federico Ambrogi ${ }^{1, \mathrm{a}}$, Sabine Kraml ${ }^{2, \mathrm{~b}}$, Suchita Kulkarni ${ }^{1, \mathrm{c}}$, Ursula Laa ${ }^{2,3, \mathrm{~d}}$, Andre Lessa ${ }^{4, \mathrm{e}}$, \\ Wolfgang Waltenberger ${ }^{1, \mathrm{f}}$ \\ ${ }^{1}$ Institut für Hochenergiephysik, Österreichische Akademie der Wissenschaften, Nikolsdorfer Gasse 18, 1050 Vienna, Austria \\ ${ }^{2}$ Laboratoire de Physique Subatomique et de Cosmologie, Université Grenoble-Alpes, CNRS/IN2P3, 53 Avenue des Martyrs, 38026 Grenoble, \\ France \\ ${ }^{3}$ LAPTh, Université Savoie Mont Blanc, CNRS, B.P.110, Annecy-le-Vieux, 74941 Annecy Cedex, France \\ ${ }^{4}$ Centro de Ciências Naturais e Humanas, Universidade Federal do ABC, Santo André, SP 09210-580, Brazil
}

Received: 17 August 2017 / Accepted: 22 February 2018 / Published online: 13 March 2018

(C) The Author(s) 2018

\begin{abstract}
We investigate to which extent the SUSY search results published by ATLAS and CMS in the context of simplified models actually cover the more realistic scenarios of a full model. Concretely, we work within the phenomenological MSSM (pMSSM) with 19 free parameters and compare the constraints obtained from SMODELS v1.1.1 with those from the ATLAS pMSSM study in arXiv: 1508.06608 . We find that about $40-45 \%$ of the points excluded by ATLAS escape the currently available simplified model constraints. For these points we identify the most relevant topologies which are not tested by the current simplified model results. In particular, we find that topologies with asymmetric branches, including 3-jet signatures from gluino-squark associated production, could be important for improving the current constraining power of simplified models results. Furthermore, for a better coverage of light stops and sbottoms, constraints for decays via heavier neutralinos and charginos, which subsequently decay visibly to the lightest neutralino are also needed.
\end{abstract}

\section{Introduction}

Simplified models [1-5] have become one of the standard methods to interpret searches for physics beyond the Standard Model (BSM). They reduce full models with dozens of particles and a plethora of parameters to subsets with just a handful of new states. The virtue of simplified model spec-

\footnotetext{
a e-mail: federico.ambrogi@oeaw.ac.at

b e-mail: sabine.kraml@1psc.in2p3.fr

c e-mail: suchita.kulkarni@oeaw.ac.at

de-mail: ursula.1aa@lpsc.in2p3.fr

e e-mail: andre.lessa@ufabc.edu.br

f e-mail: wolfgang.waltenberger@oeaw.ac.at
}

tra (SMS), namely that a full model decomposes into many different SMS, also defines their main challenge: depending on the complexity of the mass and decay patterns, a full model may not be fully reconstructed by SMS. The question that arises is to what extent full models can indeed be constrained by SMS results.

In this article, we address this question for a 19-parameter version of the minimal supersymmetric standard model, the so-called phenomenological MSSM [6], or pMSSM for short. Our work is based on the ATLAS pMSSM study [7], in which the points from an extensive pMSSM scan were tested against the constraints from 22 ATLAS searches from LHC Run 1. ATLAS made the SLHA spectra of the whole scan publicly available on HepDATA [8] together with information as regards which point is excluded by which analyses. This is extremely useful information, which we here use to test the constraining power of SMS results by means of SMODELS $[9,10]$.

SMODELS is an automatised tool for interpreting simplified model results from the LHC. It decomposes collider signatures of new physics featuring a $\mathbb{Z}_{2}$-like symmetry into simplified model topologies, using a generic procedure where each SMS is defined by the vertex structure and the Standard Model (SM) final state particles; BSM particles are described only by their masses, production cross sections and branching ratios. The weights of the various topologies, computed as production cross section times branching ratios, are then compared against a large database of experimental constraints. This procedure takes advantage of the large number of simplified models already constrained by official ATLAS and CMS results and does not require Monte Carlo event simulation, thus providing a fast way of confronting a full BSM model with the LHC constraints. Furthermore, "missing" topologies, which are not covered by any of the 
experimental constraints, are also identified and provided as an output of SMODELS.

The tool can be used for testing any BSM scenario with a $\mathbb{Z}_{2}$-like symmetry as long as all heavier odd particles (cascade-)decay promptly to the lightest one, which should be electrically and color neutral. ${ }^{1}$ It has been applied to a number of minimal and non-minimal supersymmetric (SUSY) models in [9,12-14] but may also be used for nonSUSY models; see e.g. $[15,16]$. The underlying assumption [9] that differences in the event kinematics (e.g. from different production mechanisms or from the spin of the BSM particle) do not significantly affect the signal selection efficiencies has also been investigated. For example, the effects of alternative production channels in squark simplified models were studied in [17]. The effect of a different spin structure was studied for the case of the dijet+MET final state in [15], for the dilepton+MET final state in [14] and for $t \bar{t}+\mathrm{MET}$ final states in [16]. A comprehensive study of how well a full model like the MSSM is actually covered by SMS constraints is, however, still missing. This gap we want to fill with the present paper.

We first describe the setup of the analysis in Sect. 2. Our results are presented in Sect. 3, where we discuss the exclusion obtained with SMODELS v1.1.1 as compared to ATLAS and how it is improved when including efficiency maps in addition to upper limit maps. Moreover, we discuss why a certain part of the parameter space, despite being excluded by the ATLAS study, is not excluded by (the currently available) SMS results. In particular, we analyze the importance of asymmetric decay branches and long cascade decays to understand the potential for increasing the coverage, and we point out a number of important SMS beyond those typically considered by the experimental collaborations. Conclusions are presented in Sect. 4. Appendices A and B contain useful additional material on the missing topologies discussed in the paper.

\section{Setup of the analysis}

In [7] ATLAS has analyzed in total more than 310k pMSSM parameter points with SUSY masses below $4 \mathrm{TeV}$ and a neutralino as the lightest SUSY particle (LSP). These points from an extensive scan, based on previous phenomenological studies [18-21], satisfy constraints from previous collider searches, flavor and electroweak (EW) precision measurements, cold dark matter relic density and direct dark matter searches. In addition, the mass of the light Higgs boson was required to be between 124 and $128 \mathrm{GeV}$. These points were classified into three sets according to the nature of the

\footnotetext{
1 The treatment of charged tracks is also possible in the context of simplified models [11] and will be available in future versions of SMODELS.
}

LSP: bino-like (103410 points), wino-like (80233 points) and higgsino-like (126684 points). About $40 \%$ of all these points were excluded by at least one of the 22 ATLAS Run 1 searches.

The points excluded by ATLAS are the center of interest of our study: our aim is to compare the exclusion coverage obtained using SMS results only with that from full event simulation. (In the following we mean by "coverage" the fraction of points excluded by ATLAS which is also excluded by SMODELS.) We restrict our analysis to the sets with bino-like or higgsino-like LSP, neglecting points with a wino-like LSP, as most of them lead to a displaced vertex signature, which cannot be studied with the current version of SMODELS. We further remove points from the bino- and higgsino-like LSP datasets if they contain any long-lived sparticles-this concerns, however, only a small number of points. Likewise, points which ATLAS found to be excluded only by heavy Higgs searches are also not considered here, as such searches are not treated in SMODELS for the time being. This selection leaves us with 38575 parameter points with a bino-like LSP and 45594 parameter points with a higgsino-like LSP to be tested with SMODELS.

We use the latest version of SMODELS, v1.1.1, which works with upper limit (UL) and efficiency map (EM) type results; see [10]. The cross sections for all points are calculated with the SMODELS cross section calculator interfaced to Pythia 8.2 [22,23] and NLLfast [24-31]. (The exception are the cross sections for slepton-pair production, for which we use Pythia 6.4 [22] because they are not computed correctly in Pythia 8.226.) Electroweak cross sections are thus computed at leading order while strong productions are computed at NLO+NLL order. Given the information on cross sections $(\sigma)$ and decay branching ratios (BR) in the SLHA [32] files, SMODELS computes $\sigma \times$ BR for each topology that occurs. Topologies are characterised by the SM particles originating from each vertex, and the mass vector of the SUSY particles in the decays. In order to avoid dealing with a large number of irrelevant processes, i.e. to save CPU time, topologies for which $\sigma \times \mathrm{BR}<$ sigmacut, with sigmacut $=0.03 \mathrm{fb}$, are discarded.

In addition, if the mass gap between mother and daughter particles is small, the decay products will be too soft to be detected at the LHC. This is taken care of by the so-called "mass compression" in SMODELS, discarding any SM particle coming from a vertex for which the mass splitting of the R-odd particles is less than a certain threshold. We use the default value of $5 \mathrm{GeV}$ as the minimum required mass difference for the decay products to be visible.

After the decomposition, the weights (i.e. $\sigma \times \mathrm{BR}$ ) of the SMS components of each point are rescaled by the corresponding efficiencies (see [10] for more details) and matched with the experimental results in the database. In the case of UL maps, this is a direct comparison of individual weights 
Table 1 The 22 searches considered in the ATLAS pMSSM study [7] and their correspondences in the SMODELS v1.1.1 database. A superscript * with the ID means that in addition FASTLIM EMs for a preliminary version of the analysis are included, see Table 2. The monojet results from [43] are not implemented in SMODELS because our SMS assumptions do not apply to dark matter simplified models. The analyses $[50,53,55]$ do not provide useable SMS interpretations. Finally, searches for new resonances, long-lived particles, and disappearing tracks [56-59] currently cannot be treated in the SMODELS framework

\begin{tabular}{|c|c|c|c|}
\hline Analysis & Ref. & ID & SModelS database \\
\hline \multicolumn{4}{|l|}{ Inclusive } \\
\hline 0 -lepton $+2-6$ jets $+E_{T}^{\text {miss }}$ & {$[35]$} & SUSY-2013-02* & $6 \mathrm{UL}, 2 \mathrm{EM}$ \\
\hline 0 -lepton $+7-10$ jets $+E_{T}^{\text {miss }}$ & [36] & SUSY-2013-04* & $1 \mathrm{UL}, 10 \mathrm{EM}$ \\
\hline 1-lepton + jets $+E_{T}^{\text {miss }}$ & {$[37]$} & SUSY-2013-20* & 1 UL from CONF-2013-089 [38] \\
\hline$\tau(\tau / \ell)+$ jets $+E_{T}^{\mathrm{miss}}$ & [39] & SUSY-2013-10 & n.i. \\
\hline $\mathrm{SS} / 3$-leptons + jets $+E_{T}^{\text {miss }}$ & {$[40]$} & SUSY-2013-09 & $1 \mathrm{UL}$ (+5 UL, CONF-2013-007 [41]) \\
\hline 0/1-lepton $+3 b$-jets $+E_{T}^{\text {miss }}$ & {$[42]$} & SUSY-2013-18* & $2 \mathrm{UL}, 2 \mathrm{EM}$ \\
\hline Monojet & {$[43]$} & - & - (but monojet stop; see below) \\
\hline \multicolumn{4}{|l|}{ Third generation } \\
\hline 0-lepton stop & {$[44]$} & SUSY-2013-16* & $1 \mathrm{UL}, 1 \mathrm{EM}$ \\
\hline 1-lepton stop & {$[45]$} & SUSY-2013-15* & $1 \mathrm{UL}, 1 \mathrm{EM}$ \\
\hline 2-leptons stop & [46] & SUSY-2013-19* & $2 \mathrm{UL}$ \\
\hline Monojet stop & {$[47]$} & SUSY-2013-21 & $4 \mathrm{EM}$ \\
\hline Stop with $Z$ boson & [48] & SUSY-2013-08 & $1 \mathrm{UL}$ \\
\hline $2 b$-jets $+E_{T}^{\text {miss }}$ & [49] & SUSY-2013-05* & $3 \mathrm{UL}, 1 \mathrm{EM}$ \\
\hline$t b+E_{T}^{\text {miss }}$, stop & {$[50]$} & SUSY-2014-07 & - \\
\hline \multicolumn{4}{|l|}{ Electroweak } \\
\hline$\ell h$ & {$[51]$} & SUSY-2013-23* & $1 \mathrm{UL}$ \\
\hline 2-leptons & {$[52]$} & SUSY-2013-11 & $4 \mathrm{UL}, 4 \mathrm{EM}$ \\
\hline $2-\tau$ & {$[53]$} & SUSY-2013-14 & - \\
\hline 3-leptons & {$[54]$} & SUSY-2013-12 & $5 \mathrm{UL}$ \\
\hline 4-leptons & {$[55]$} & SUSY-2013-13 & - \\
\hline Disappearing Track & {$[56]$} & SUSY-2013-01 & n.a. \\
\hline \multicolumn{4}{|l|}{ Other } \\
\hline Long-lived particle & {$[57,58]$} & - & n.a. \\
\hline$H / A \rightarrow \tau^{+} \tau^{-}$ & [59] & - & n.a. \\
\hline
\end{tabular}

and the cross section upper limit for a given simplified model component or topology. In the case of EMs, the weights of several topologies can be combined and may contribute to a specific signal region of a given analysis; it is then the combined signal cross section for the most sensitive signal region (i.e. the signal region with the best expected limit) which is compared against the experimental limit. Hence using efficiency maps can significantly improve the constraining power of simplified models. See the SMODELS v1.1.1 manual [10] for a detailed explanation of the procedure.

For a fair comparison with [7], we employ only the $8 \mathrm{TeV}$ results in the v1.1.1 database. In order to maximize the coverage by SMS, we consider, however, also CMS $8 \mathrm{TeV}$ results, as they may give complementary constraints. This is justified because ATLAS and CMS SUSY searches largely consider the same final states and have very similar reach. We also note that the official ATLAS and CMS Run 1 results available in SMODELS were augmented with several 'home-grown' EMs in the v1.1.1 database to increase the coverage, and we further extend this database with FASTLIM-1.0 [33] EMs as explained in [10]. The complete list of analyses and results included in the v1.1.1 database can be consulted at [34].
A comparison of the analyses considered in [7] and the SMS results included in SMODELS v1.1.1 is given in Table 1. The analyses covered by the FASTLIM EMs are listed in Table 2. Here note that in SMODELS v1.1.1 efficiencies with a relative statistical uncertainty greater than $25 \%$ are set to zero and, moreover, zero-only EMs are discarded per default. Therefore, from the 264 EMs of FASTLIM-1.0, which are based on 11 ATLAS conference notes, used in practice are 163 EMs from 9 conference notes. The CMS analyses included in the v1.1.1 database are listed in Table 3.

SMODELS reports its results in the form of $r$-values, defined as the ratio of the theory prediction over the observed 95\% confidence level (CL) upper limit, for each experimental constraint that is matched in the database. We consider as excluded all points for which at least one $r$-value equals or exceeds unity $\left(r_{\max } \geq 1\right){ }^{2}$ Points which are not excluded

\footnotetext{
$\overline{2}$ We note that for staying strictly at $95 \%$ CL, one should use only the $r$-value of the most sensitive analysis. This is, however, not feasible because for many UL-type results the expected limits are not publicly available.
} 
Table 2 Analyses covered by the FASTLIM [33] EMs converted to the SMODELS format. For each analysis, FASTLIM considers 24 topologies covering stop-, sbottom- and gluino-pair production with direct or cascade decays to a higgsino LSP, inspired by the idea of "natural SUSY". As explained in the text, efficiencies with uncertainties $>25 \%$ are set to zero, so in practice we use 163 of the FASTLIMEMs

\begin{tabular}{lll}
\hline Analysis & Ref. & ID \\
\hline Incl. & & \\
0-lepton + 2-6 jets $+E_{T}^{\text {miss }}$ & {$[60]$} & ATLAS-CONF-2013-047 \\
0-lepton + 7-10 jets $+E_{T}^{\text {miss }}$ & {$[61]$} & ATLAS-CONF-2013-054 \\
1-lepton + jets $+E_{T}^{\text {miss }}$ & {$[62]$} & ATLAS-CONF-2013-062 \\
0/1-lepton + 3b-jets $+E_{T}^{\text {miss }}$ & {$[63]$} & ATLAS-CONF-2013-061 \\
Third gen. & & \\
0-lepton stop & {$[64]$} & ATLAS-CONF-2013-024 \\
1-lepton stop & {$[65]$} & ATLAS-CONF-2013-037 \\
2-leptons stop & {$[66]$} & ATLAS-CONF-2013-048 \\
2b-jets $+E_{T}^{\text {miss }}$ & {$[67]$} & ATLAS-CONF-2013-053 \\
EW & & \\
$\ell h$ & {$[68]$} & ATLAS-CONF-2013-093 \\
\hline
\end{tabular}

$\left(r_{\max }<1\right)$ are further studied using the SMODELS coverage module (see section 3.5 in [10]).

\section{Exclusion compared to ATLAS}

As a first overview of our results, we list in Table 4 the total number of points studied, the number of points that can be excluded by SMODELS ( $r_{\max } \geq 1$ ) when using only the UL results in the database, and the number of points that can be excluded when using the full $8 \mathrm{TeV}$ database, that is, including EM results. We see that the coverage of bino-like LSP scenarios can be improved by using EMs, increasing from $44 \%$ (UL results only) to $55 \%$ (full database). Similarly, the coverage for the higgsino-like LSP scenarios is improved from 55 to $63 \%$.

The improvement in coverage due to EMs largely happens for light to intermediate gluino masses, as illustrated in Fig. 1. These scenarios benefit from the fact that EMs allow us to combine the signal for all topologies contributing to the same signal region before comparing against an overall cross
Table $3 \mathrm{CMS} 8 \mathrm{TeV}$ results included in the SMODELS v1.1.1 database and used in addition to the ATLAS results in Tables 1 and 2

\begin{tabular}{llll}
\hline Analysis & Ref. & ID & SModelS database \\
\hline Gluino, Squark & & & \\
Jets $+E_{T}^{\text {miss }}, \alpha_{T}$ & {$[69]$} & SUS-12-028 & 4 UL \\
$3\left(1 b\right.$-)jets $+E_{T}^{\text {miss }}$ & {$[70]$} & SUS-12-024 & 2 UL, 3 EM \\
Jet multiplicity $+H_{T}^{\text {miss }}$ & {$[71]$} & SUS-13-012 & 4 UL, 20 EM \\
$\geq 2$ jets $+E_{T}^{\text {miss }}, M_{T 2}$ & {$[72]$} & SUS-13-019 & 8 UL \\
$\geq 1 b+E_{T}^{\text {miss }}$, Razor & {$[73]$} & SUS-13-004 & 5 UL \\
1 lepton $+\geq 2 b$-jets $+E_{T}^{\text {miss }}$ & {$[74]$} & SUS-13-007 & 3 UL, EM \\
2 OS lept. $+\geq 4\left(2 b\right.$-)jets $+E_{T}^{\text {miss }}$ & {$[75]$} & PAS-SUS-13-016 & 2 UL \\
2 SS leptons $+b$-jets $+E_{T}^{\text {miss }}$ & {$[76]$} & SUS-13-013 & 4 UL, 2 EM \\
$b$-jets $+4 W \mathrm{~s}+E_{T}^{\text {miss }}$ & {$[77]$} & SUS-14-010 & 2 UL \\
Third gen. & & & \\
0 lepton $+\geq 5\left(1 b\right.$-)jets $+E_{T}^{\text {miss }}$ & {$[78]$} & PAS-SUS-13-015 & 2 EM \\
0 lepton $+\geq 6\left(1 b\right.$-)jets $+E_{T}^{\text {miss }}$ & {$[79]$} & PAS-SUS-13-023 & 4 UL \\
1 lepton $+\geq 4\left(1 b\right.$-)jets $+E_{T}^{\text {miss }}$ & {$[80]$} & SUS-13-011 & 4 UL, 2 EM \\
$b$-jets $+E_{T}^{\text {miss }}$ & {$[81]$} & PAS-SUS-13-018 & 1 UL \\
Soft leptons, few jets $+E_{T}^{\text {miss }}$ & {$[82]$} & SUS-14-021 & 2 UL \\
EW & & & \\
Multi-leptons $+E_{T}^{\text {miss }}$ & {$[83]$} & SUS-13-006 & 6 UL \\
\hline
\end{tabular}

Table 4 Summary of results, listing the number of ATLAS-excluded pMSSM points tested in this study, the number of points excluded by SMODELS when using UL-type results only, and the number of points excluded when using the full $8 \mathrm{TeV}$ database including EM-type results

\begin{tabular}{lll}
\hline & Bino-like LSP & Higgsino-like LSP \\
\hline Total number of points & 38575 & 45594 \\
Number of points excluded_UL results only & 16957 & 25024 \\
Number of points excluded_full database & 21151 & 28669 \\
\hline
\end{tabular}




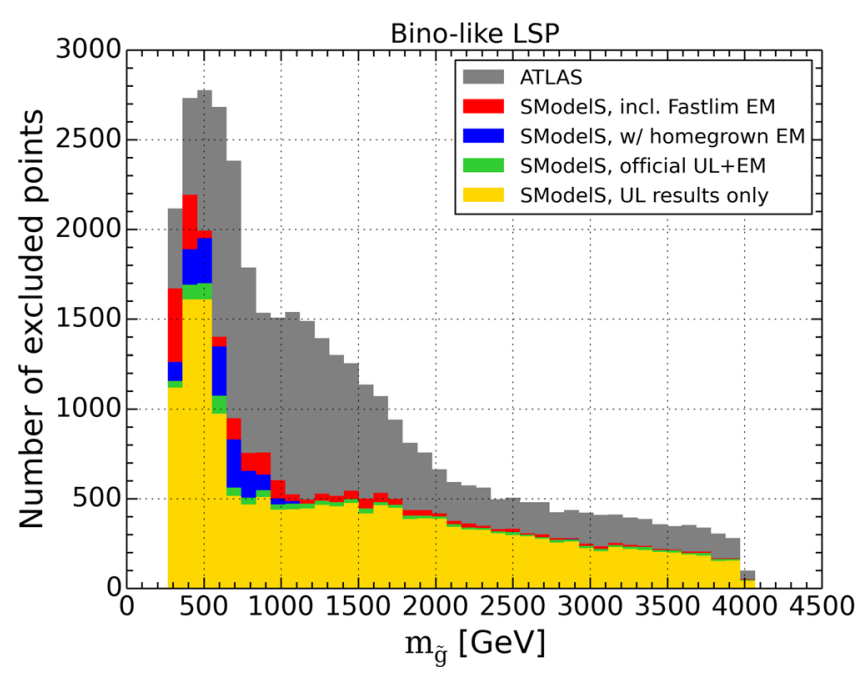

Fig. 1 Number of points excluded by SMODELS using only UL results (in yellow), adding official EM results (in green), adding 'home-grown' EMs (in blue) and finally adding also FASTLIM EMs (in red). For ref-

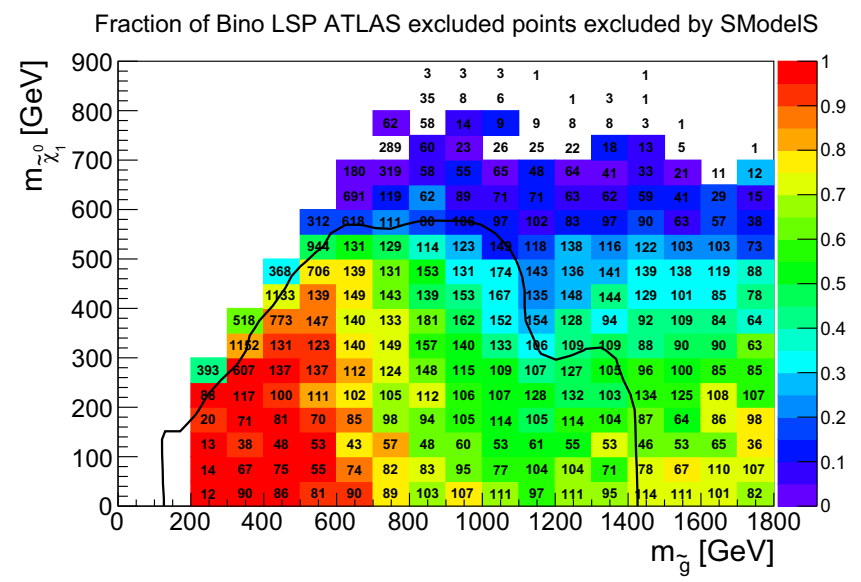

Fig. 2 Coverage in the gluino vs. neutralino mass plane, for gluino masses up to $2 \mathrm{TeV}$, for bino-like LSP scenarios (left) and higgsino-like LSP scenarios (right). The color code indicates the fraction of points

section limit, hence increasing the constraining power. Moreover, some asymmetric topologies are included in the EMtype results (from FASTLIM) but not in the UL-type results in the database. Figure 1 also shows the importance of the FASTLIM and our 'home-grown' EMs with respect to the official ATLAS and CMS SMS results. We note that the FASTLIM maps are particularly relevant for constraining gluinos in the intermediate mass range decaying to higgsino-like EW-inos, which is typical for the natural SUSY case they have been derived for. In numbers, official UL and EM results exclude $46 \%(56 \%)$ of the bino-LSP (higgsino-LSP) points, which improves to $50 \%$ (57\%) when adding our 'home-grown' EMs, and to the above-mentioned 55\% (63\%) when including in addition FASTLIM results. In the following, we discuss

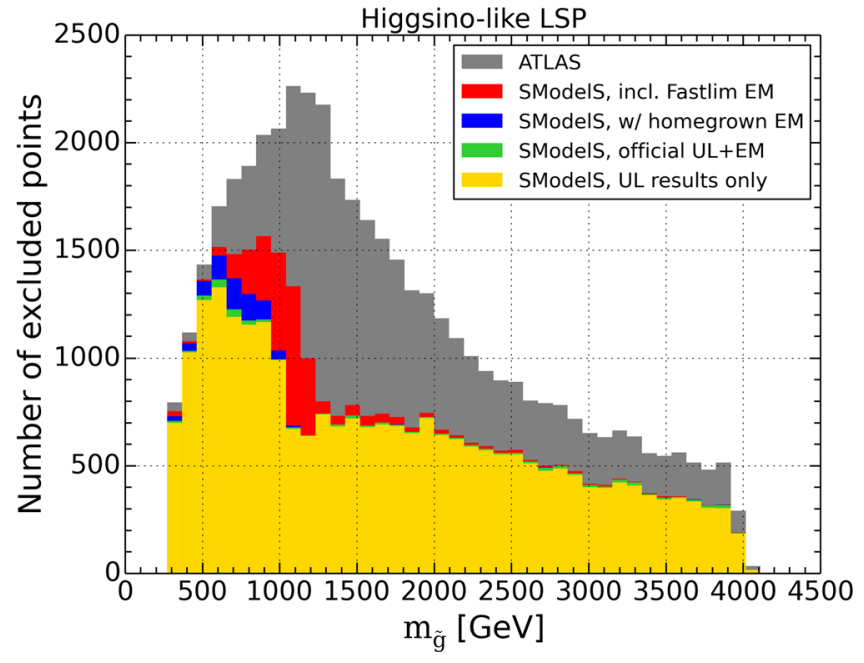

erence the total number of ATLAS-excluded points is also shown (in gray). On the left for bino-like LSP and on the right for higgsino-like LSP

Fraction of Higgsino LSP ATLAS excluded points excluded by SModelS

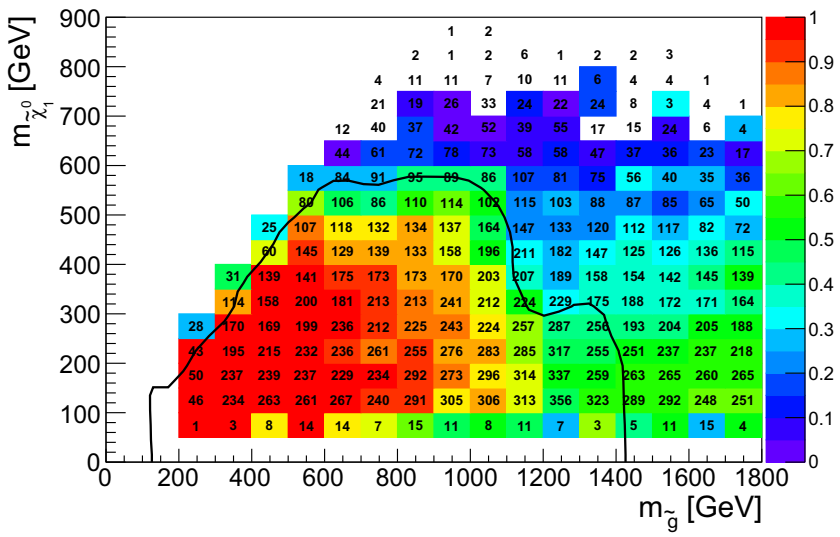

excluded by SMODELS, the text gives the total number of points tested in each bin. For comparison, the 95\% CL exclusion line for the $\tilde{g} \rightarrow q q \tilde{\chi}_{1}^{0}$ simplified model from [35] is drawn in black

in some detail why still a large fraction of points escapes exclusion by SMS results and how the coverage could be improved.

\subsection{Gluinos}

It is striking that there are many points with light gluinos which cannot be excluded by the SMS results in the SMODELS database. To understand this better we show in Fig. 2 the coverage in the gluino vs. neutralino mass plane. For comparison with the "naive" SMS expectation, the exclusion line obtained in [35] for a simplified model where pair-produced gluinos decay exclusively as $\tilde{g} \rightarrow q q \tilde{\chi}_{1}^{0}$ is also drawn in Fig. 2. We see that light gluinos escape SMS limits espe- 

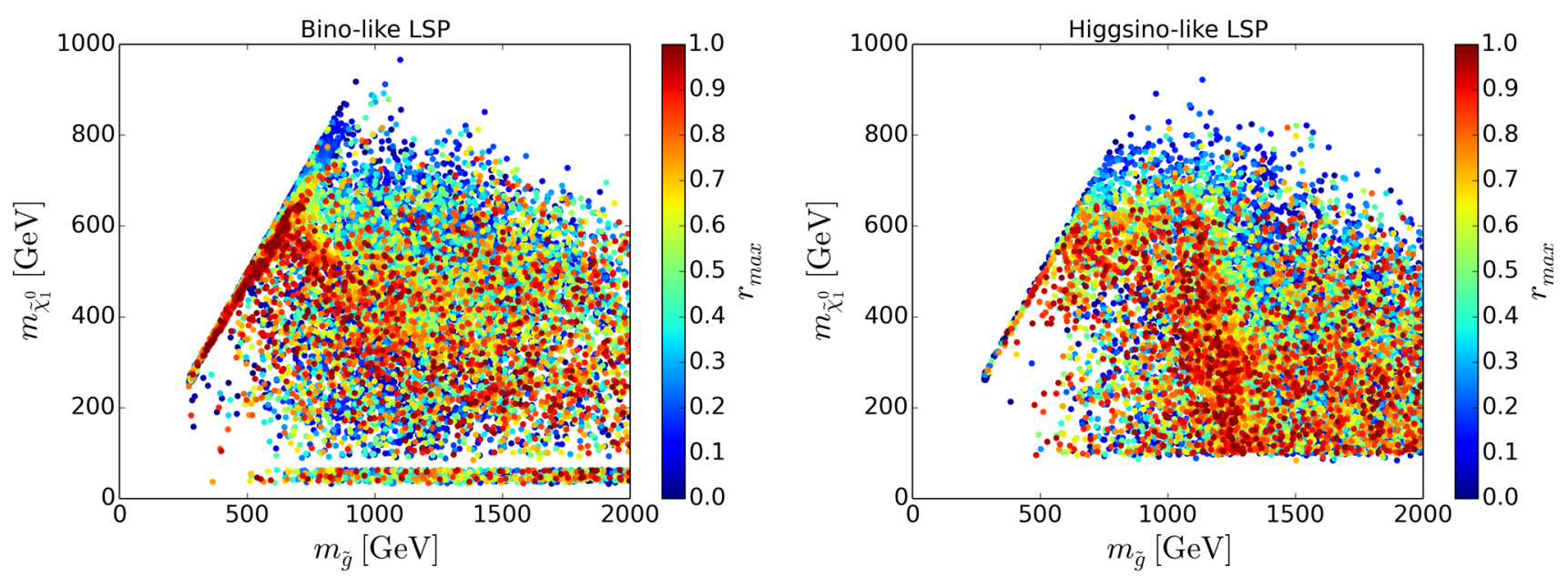

Fig. 3 Maximum $r$ value reported by SModELS for allowed points, for gluino masses up to 2 TeV, for bino-like LSP scenarios (left) and higgsinolike LSP scenarios (right). Points are sorted from low to high $r$-values, with the highest values of $r$ shown on top

cially in the compressed region where monojet-type searches become important. This is in agreement with the simplified model exclusion line. Moreover, while the coverage is good for very light gluinos up to about $600 \mathrm{GeV}$, it drops for intermediate gluino masses around $1 \mathrm{TeV}$ and higher, as can also be observed in Fig. 1. This is particularly pronounced in the bino-like LSP scenario. Concretely, the coverage of binolike LSP scenarios is $80 \%$ when considering only points with light gluinos $\left(m_{\tilde{g}}<600 \mathrm{GeV}\right)$, but drops to $60 \%$ when considering all points with $m_{\tilde{g}}<1400 \mathrm{GeV}$. Similarly, the coverage of higgsino-like LSP scenarios drops from $97 \%$ $\left(m_{\tilde{g}}<600 \mathrm{GeV}\right)$ to $74 \%\left(m_{\tilde{g}}<1400 \mathrm{GeV}\right)$. Note that for bino-like LSP scenarios light gluinos are mainly found in the compressed region $\left(m_{\tilde{g}}-m_{\tilde{\chi}_{1}^{0}}<100 \mathrm{GeV}\right)$, where the bins contain a large number of model points. This is not the case for higgsino-like LSP scenarios.

The somewhat better coverage of non-compressed sub$\mathrm{TeV}$ gluinos in the higgsino-like LSP set can be understood as follows. In the case of a bino-like LSP, unless the gluinoLSP mass difference is small, direct decays into the LSP often have only $30 \%$ or less branching ratio. Decays into winoor higgsino-like states are often more important, leading to cascade decays into the LSP and to asymmetric branches with different final states and, possibly, different intermediate masses. ${ }^{3}$ This reduces the fraction of gluino signatures covered by SMS results, and as the total cross section reduces with increasing gluino mass, the fraction that can be constrained is no longer large enough to exclude the point. For higgsino-like LSP scenarios, on the other hand, the second neutralino $\tilde{\chi}_{2}^{0}$ as well as the lighter chargino $\tilde{\chi}_{1}^{ \pm}$are nearly degenerate with the LSP, and their decay can often be mass

\footnotetext{
3 Asymmetric branches can occur from pair production when the two initially produced SUSY particles undergo different decays, or from associated production of two different SUSY particles.
}

compressed in SMODELS. In this case, contributions from $\tilde{g} \rightarrow q q^{\prime} \tilde{\chi}_{1}^{ \pm}, q q \tilde{\chi}_{2}^{0}$ and $q q \tilde{\chi}_{1}^{0}$ can be summed up, which explains the better coverage of light gluinos in the higgsinoLSP case already by UL results seen in Fig. 1. Moreover, gluino decays into third generation are often dominant in the higgsino-LSP case, leading to a mix of final states $(4 b$, $4 t, 2 b 2 t, 3 b 1 t, 1 b 3 t)$ which can in part be covered by the FASTLIM EMs.

Another important consideration is how far the points which escape the SMODELS exclusion are from becoming excluded. Uncertainties inherent to the SMODELS approach and the fact that we used $\mathrm{LO}$ cross sections for EW process (while ATLAS used NLO values) can reduce the exclusion reach. In Fig. 3 we show the maximum $r$ values found for points escaping exclusion by SMODELS. We see that many points, especially in the region of intermediate gluino masses and in the more compressed region, are in fact close to the exclusion limit. We therefore expect that the coverage can be considerably improved by adding additional EMs, thus allowing to test a larger fraction of the total cross section. Furthermore, we find that $10 \%$ of bino-like LSP scenarios and $12 \%$ of higgsino-like LSP scenarios have $0.8<r_{\max }<1.2$, which allows a rough estimate of the uncertainties involved in the exclusion. (The overall systematic uncertainty is estimated to be at the level of $20 \%$ [10].) In turn, we find $r_{\max }>1.2$ for $50 \%$ of bino-like LSP and $58 \%$ of higgsinolike LSP scenarios.

To understand the possibilities of further improving the coverage, without going into details as regards the specific missing topologies, ${ }^{4}$ we show in Figs. 4 and 5 the rela-

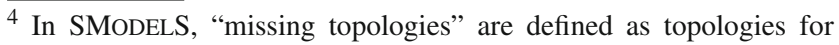
which no experimental result is available in the database.
} 


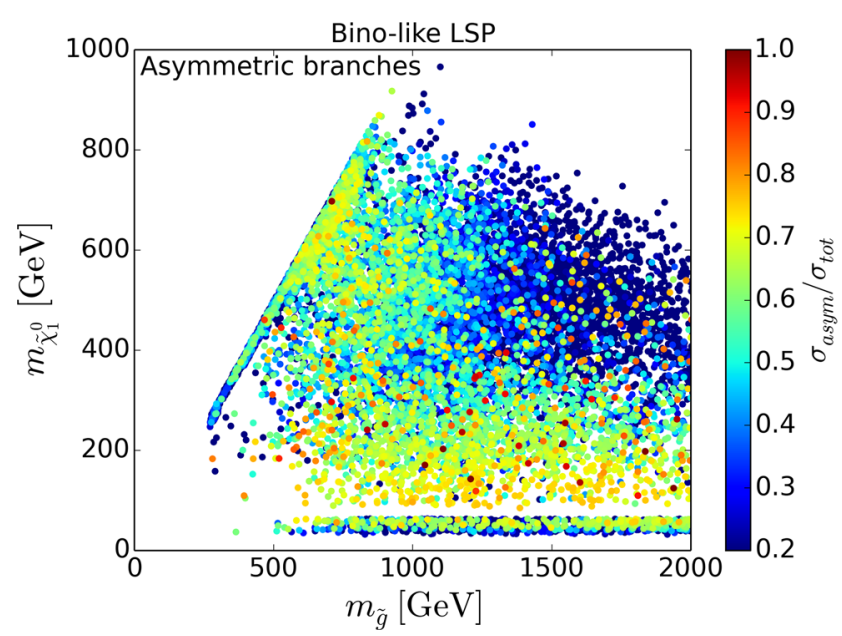

Fig. 4 Relative cross section in unconstrained decays with asymmetric branches (left) and long cascade decays (right), for scenarios with a bino-like LSP. Here the total cross section $\sigma_{t o t}$ refers to the full $8 \mathrm{TeV}$

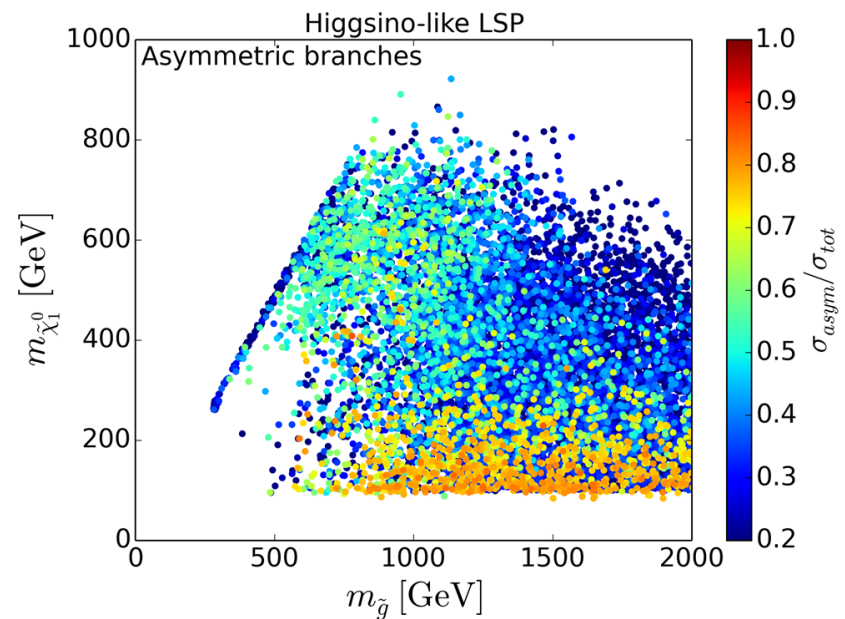

Fig. 5 Same as Fig. 4 but for points with a higgsino-like LSP

tive cross sections of SMODELS-allowed ${ }^{5}$ points which go into missing topologies with asymmetric branches (left) or long cascade decays (right), for bino-like LSP scenarios and higgsino-like LSP scenarios, respectively. In this classification, asymmetric branch topologies have at most one intermediate odd particle in each branch, so that the number of new particles and mass parameters still is sufficiently small for a viable SMS interpretation. On the other hand, as long cascade decays we define decay chains with two or more intermediate odd particles and we no longer consider a simplified model description viable. We see that in fact topologies with asymmetric decay branches are important for a large number of points for both bino- and higgsino-like LSP scenarios, whereas long cascade decay topologies are dominant only

\footnotetext{
5 We define "SMODELS-allowed" as "excluded by ATLAS but not excluded by SMODELS".
}

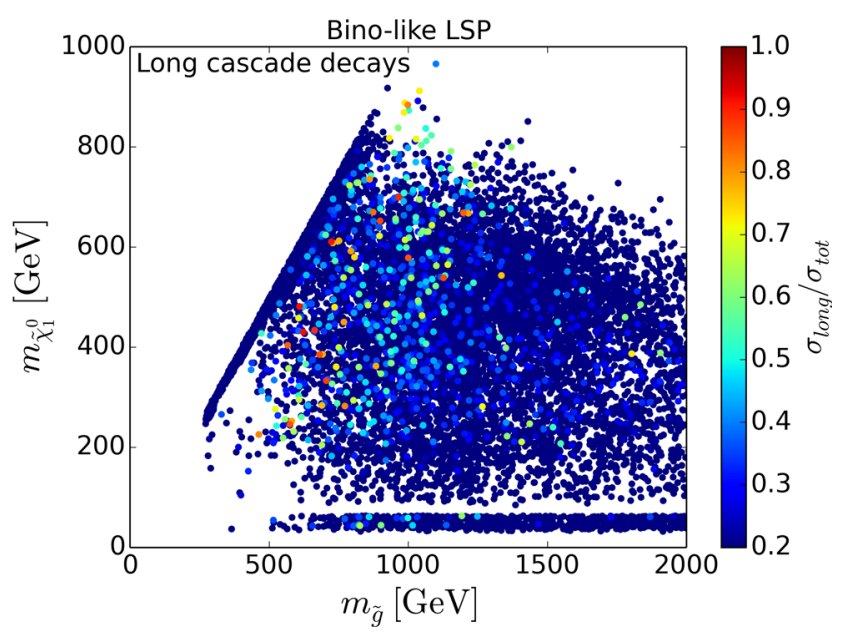

SUSY cross section. Only SMoDELS-allowed points with total cross section larger than $10 \mathrm{fb}$ are considered

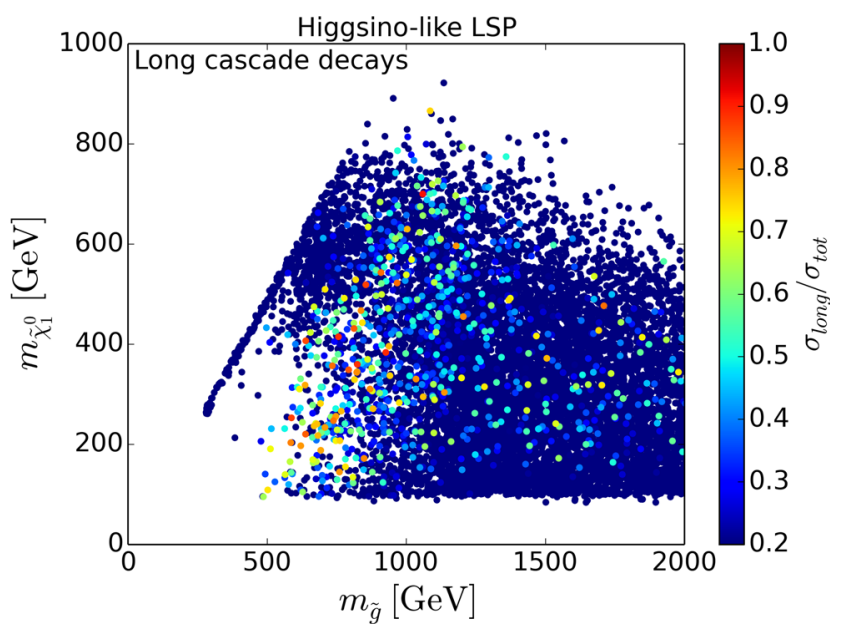

in a few cases. Therefore inclusion of additional asymmetric topologies should have a significant impact on the SMS coverage.

A particularly important missing topology with asymmetric branches arises from gluino-squark associate production, giving a 3 jets $+E_{T}^{\text {miss }}$ final state. This is important in particular when the light-flavor squarks are highly split and the gluino can decay to a single on-shell squark. The relevant process is $p p \rightarrow \tilde{g} \tilde{q}$ followed by $\tilde{q} \rightarrow q \tilde{\chi}_{1}^{0}$ on one branch and $\tilde{g} \rightarrow q \tilde{q} \rightarrow q \bar{q} \tilde{\chi}_{1}^{0}$ on the other branch. The same topology is possible when gluinos are lighter than all squarks and decay dominantly via a loop decay to a gluon and the neutralino LSP. In this case we have $p p \rightarrow \tilde{g} \tilde{q}$ followed by $\tilde{g} \rightarrow g \tilde{\chi}_{1}^{0}$ on one branch and $\tilde{q} \rightarrow q \tilde{g} \rightarrow q g \tilde{\chi}_{1}^{0}$ on the other. Figure 6 shows the cross section of this topology in the plane of gluinos mass versus mass of the lightest squark. Note that searches for gluino-squark production are typically inter- 

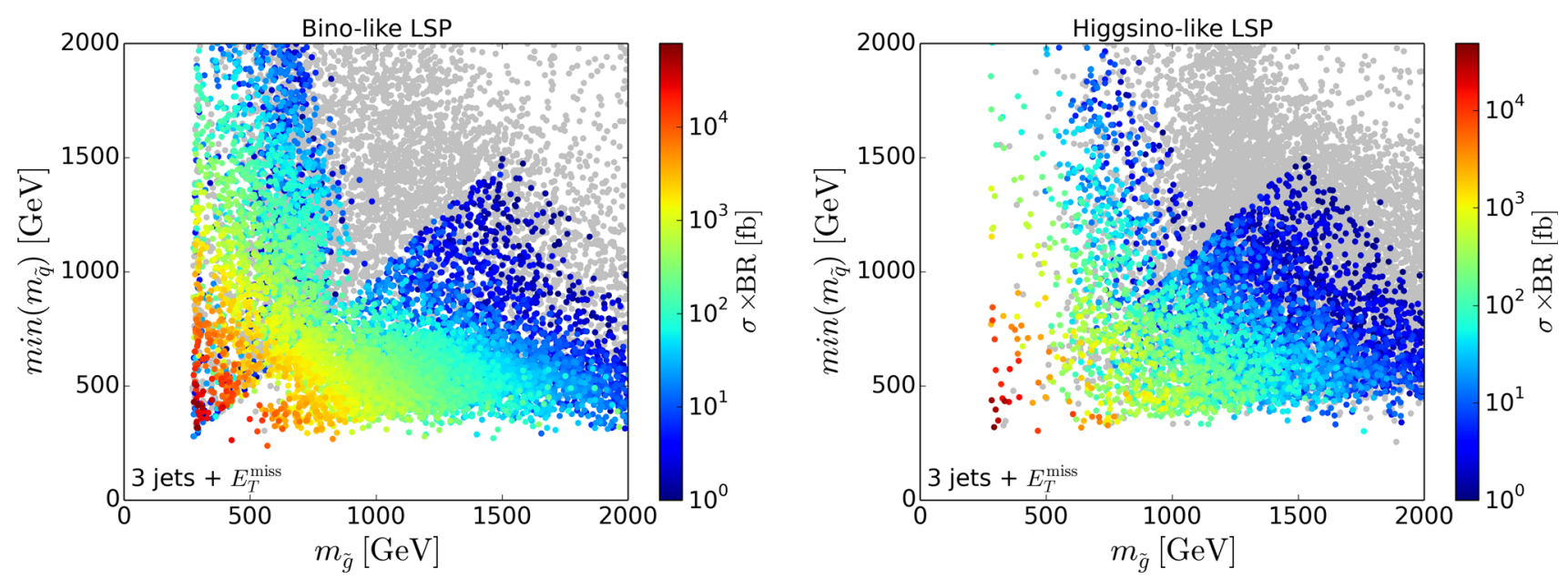

Fig. 6 Cross section for the $\tilde{g} \tilde{q} \rightarrow 3$ jets $+E_{T}^{\text {miss }}$ missing topology in the gluino vs. squark mass plane, for bino-like LSP (left) and higgsino-like LSP (right). Only SMODELS-allowed points are considered

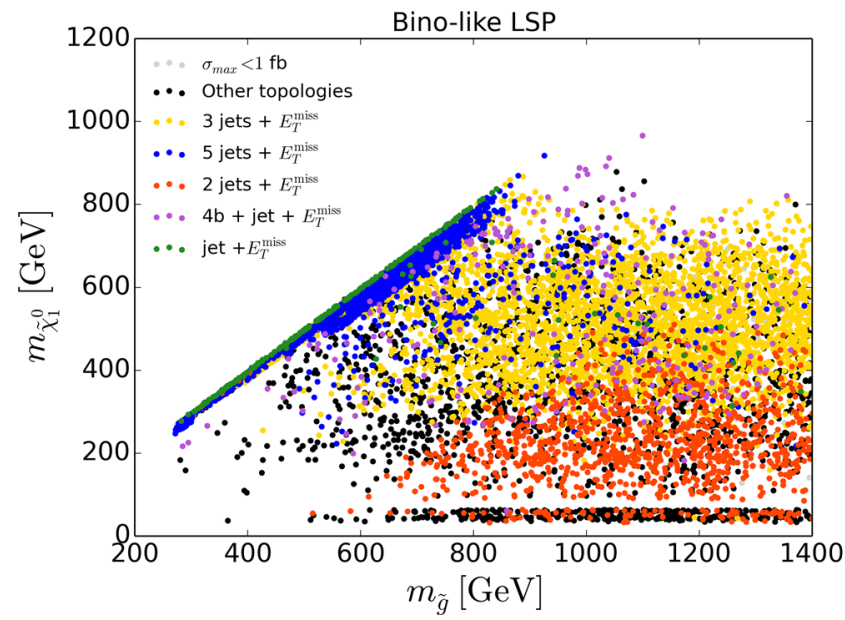

Fig. 7 Most important missing topologies for SMODELS-allowed points with light gluinos. The legend lists, from top to bottom, the missing topologies with highest cross sections ordered by their by fre-

preted either in a simplified model where gluinos and squarks are (nearly) mass-degenerate, or in a minimal gluino-squark model where all production processes-gluino pairs, squark pairs, and gluino-squark associated production-are combined [35]. Such results cannot be used for reinterpretation in generic scenarios where typically the gluino mass differs from the squark masses, and where the relative importance of the various production and decay channels will be different from the minimal gluino-squark model description.

The importance of the 3 jets $+E_{T}^{\text {miss }}$ topology is corroborated in Fig. 7, which shows the five most important missing topologies for points with light gluinos below $1.5 \mathrm{TeV}^{6}{ }^{\mathrm{T}}$ The

\footnotetext{
${ }^{6}$ For this classification, we first select for each allowed point the missing topology with the highest cross section. These are then sorted by frequency of occurrence in the mass range considered.
}

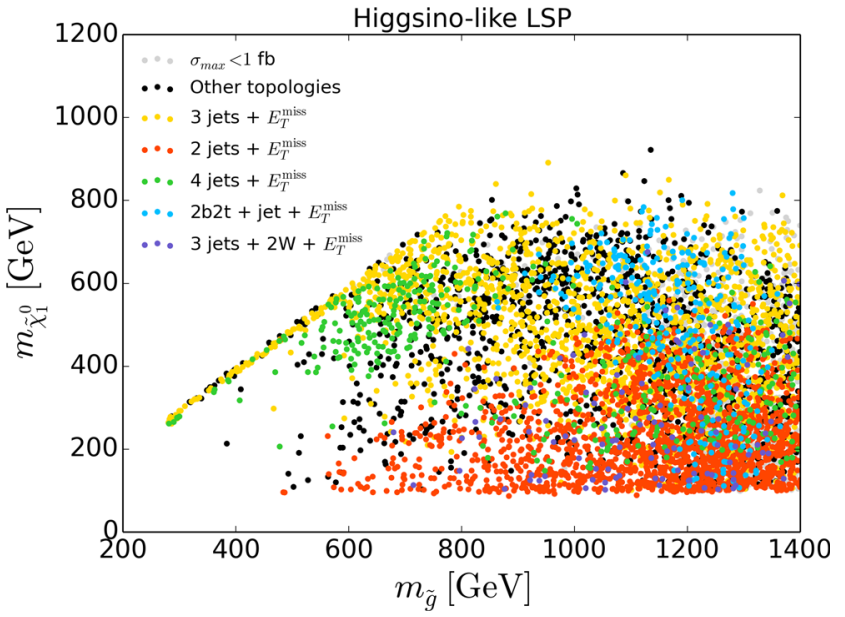

quency of occurrence (points in color). The relevant diagrams, SUSY processes and labeling in SMODELS notation are given in Appendix A

leading missing topology for both the bino- and the higgsinoLSP datasets is indeed 3 jets $+E_{T}^{\text {miss }}$ from gluino-squark associated production as discussed above; see the yellow points in Fig. 7, which cover a wide range of gluino and LSP masses. Gluino-squark associated production also leads to the 5 jets $+E_{T}^{\text {miss }}$ missing topology; in this case all squarks are heavier than the gluino and decay via $\tilde{q} \rightarrow q \tilde{g}$, and the gluino then decays further to two jets and the $\tilde{\chi}_{1}^{0}$. This is the dominant missing topology for compressed gluino and neutralino masses in the bino-like LSP case; see the blue points in the left panel of Fig. 7. When compressing the gluino and LSP masses even further, such that the gluino decay is not visible any more, this gives jet $+E_{T}^{\text {miss }}$ (dark green points), which is, however, a rather fine-tuned situation in the pMSSM and thus occurs much less often. 


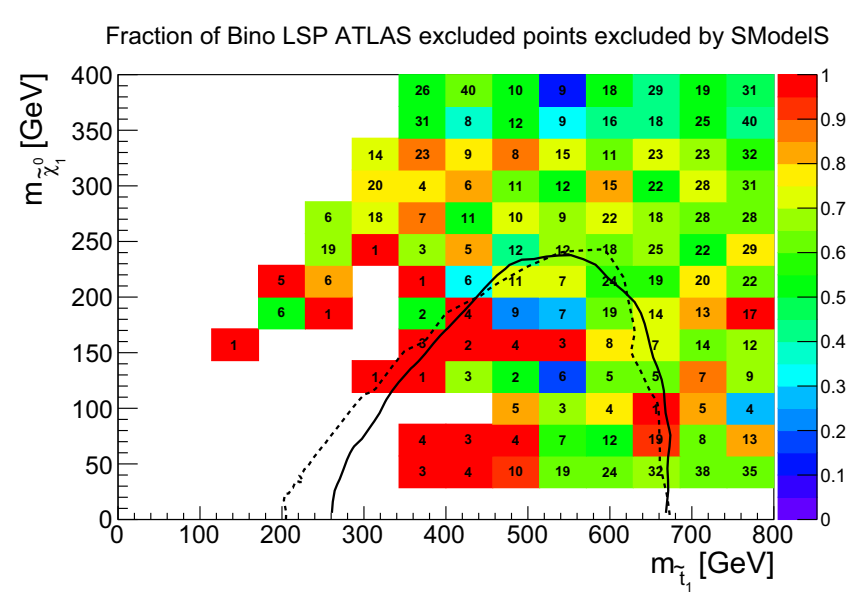

Fig. 8 Coverage in the stop vs. neutralino mass plane, for $\tilde{t}_{1}$ masses up to $800 \mathrm{GeV}$, for bino-like LSP scenarios (left) and higgsino-like LSP scenarios (right). The color code indicates the fraction of points excluded by SMODELS as compared to ATLAS, while the text gives

Also noteworthy are the orange points, which denote an asymmetric 2 jets $+E_{T}^{\text {miss }}$ topology with the two jets on one branch and nothing on the other branch. This can come from $\tilde{\chi}_{1}^{0} \tilde{\chi}_{i \neq 1}^{0}, \tilde{\chi}_{1}^{0} \tilde{\chi}_{1,2}^{ \pm}$or $\tilde{\chi}_{1}^{0} \tilde{g}$ associated production. While EW-ino and $\tilde{\chi}_{1}^{0} \tilde{g}$ production can have comparable cross sections, the latter process is often disregarded. (The same topology can also arise from gluino-squark associated production when $\tilde{g} \rightarrow q \bar{q} \tilde{\chi}_{1}^{0}$ and the $\tilde{q}$ decay is "invisible" because of mass compression with the LSP.) Other topologies like $2 b 2 t+\mathrm{jet}+E_{T}^{\text {miss }}\left(\right.$ from $p p \rightarrow \tilde{g} \tilde{q}, \tilde{q} \rightarrow q \tilde{g}, \tilde{g} \rightarrow t b \chi_{1}^{+}$in the higgsino-LSP case) or long cascades with $4 b+$ jet $+E_{T}^{\text {miss }}$ or 3 jets $+2 W+E_{T}^{\text {miss }}$ also show up in Fig. 7, but they are much less often the missing topology with highest cross section. The corresponding diagrams, SUSY processes and labeling in SMODELS notation can be found in Appendix A.

We note that all these missing topologies could be constrained from the jets $+E_{T}^{\text {miss }}$ searches, if the appropriate SMS interpretations were available. For instance, a limit of $40,20,10 \mathrm{fb}$ on the 3 jets $+E_{T}^{\text {miss }}$ missing topology cross section would exclude additional 4846, 5799, $6599(1377,1948$, 2637) points of the bino-like (higgsino-like) LSP dataset. We have explicitly checked a couple of representative SMODELSallowed points with a high 3 jets $+E_{T}^{\text {miss }}$ cross section and verified that including the efficiencies for the relevant gluinosquark simplified model would indeed exclude these points. A specific example is provided in Appendix B.

\subsection{Third generation}

Apart from gluinos and squarks, which may be regarded as the primary (and easiest) targets of the SUSY searches, searches for stops and sbottoms are of particular interest. The coverage obtained by SMODELS in the stop vs. neutralino and
Fraction of Higgsino LSP ATLAS excluded points excluded by SModelS

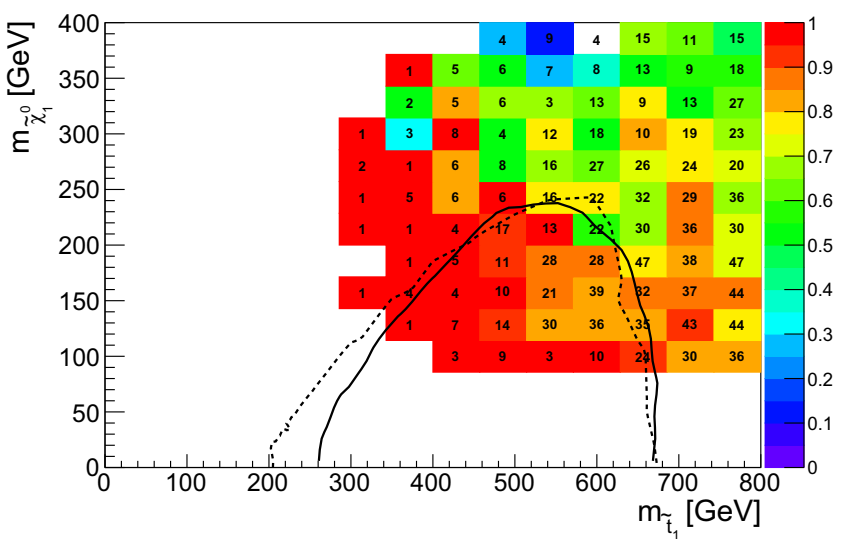

the total number of points tested in each bin. For comparison, the black lines are the $95 \% \mathrm{CL}$ exclusion curves for the $\tilde{t}_{1} \rightarrow t \tilde{\chi}_{1}^{0}$ simplified model from [44] (0-lepton mode, full line) and [45] (1-lepton mode, dashed line)

sbottom vs. neutralino mass planes is shown in Figs. 8 and 9. We also show the official exclusion curves for the $\tilde{t}_{1} \rightarrow t \tilde{\chi}_{1}^{0}$ and $\tilde{b}_{1} \rightarrow b \tilde{\chi}_{1}^{0}$ simplified models from [44,45,49], to help identify the region expected to be excluded by stop or sbottom production only.

For stops, we observe an excellent coverage in the higgsino-LSP case when compared to the official exclusion curves. (A slightly stronger exclusion is obtained by the combination of the 0-lepton and 1-lepton analyses [50], but no UL maps are available for the combination.) Contrary to the gluino case, the stop exclusion is not driven by EM results but by the UL maps for $t \bar{t}+E_{T}^{\text {miss }}$ and $b \bar{b}+E_{T}^{\text {miss }}$ final states (mostly because not so many different EMs are available for stops and sbottoms). Points outside the naive SMS exclusion line are excluded by other searches or because of light sbottoms which also contribute to the signal. In the bino-LSP case, on the other hand, light stops in the 500 $650 \mathrm{GeV}$ mass range often escape exclusion by SMS results. This is mostly because they share out their branching ratios in $\tilde{t}_{1} \rightarrow t \tilde{\chi}_{2}^{0} \rightarrow t Z \tilde{\chi}_{1}^{0}$ and $\tilde{t}_{1} \rightarrow b \tilde{\chi}_{1}^{+} \rightarrow b W \tilde{\chi}_{1}^{0}$ cascade decays. While we do have EMs for a so-called T6bbWW simplified model, i.e. a $2 b 2 W+E_{T}^{\text {miss }}$ final state originating from both stops decaying via an intermediate chargino, the equivalent topologies for one or both stops decaying via an intermediate neutralino (e.g., $t b W Z+E_{T}^{\text {miss }}$ and $2 t 2 Z+E_{T}^{\text {miss }}$ final states) are missing. Including EMs for these topologies for a variety of intermediate $\tilde{\chi}_{2}^{0}$ and $\tilde{\chi}_{1}^{ \pm}$masses would certainly allow us to get closer to the ATLAS exclusion. ${ }^{7}$ Notice, however, that for light stops we are dealing with small numbers

\footnotetext{
${ }^{7}$ Note that for cascade decays via an intermediate sparticle, it is important to have several mass planes in order to be able to interpolate in all dimensions of the SMS; see also Appendix C of [10].
} 


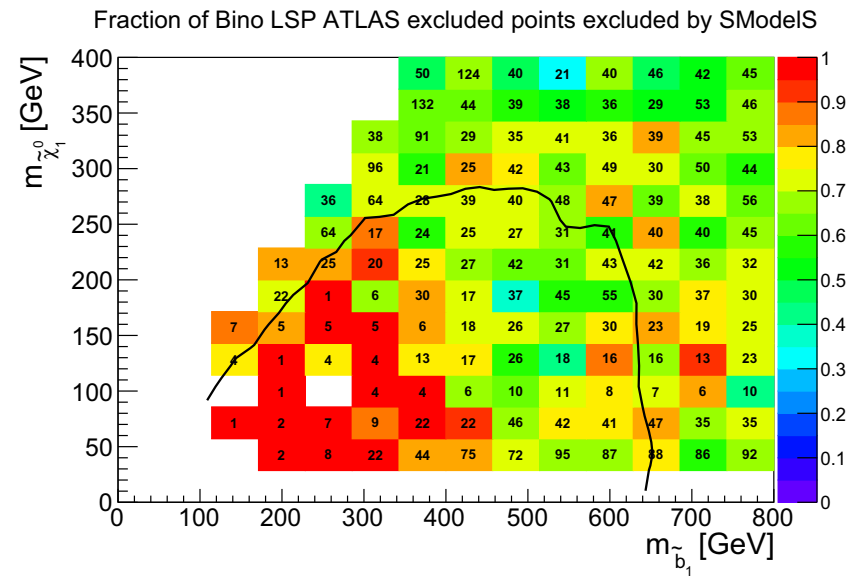

Fig. 9 Coverage in the sbottom vs. neutralino mass plane, for $\tilde{b}_{1}$ masses up to $800 \mathrm{GeV}$, for bino-like LSP scenarios (left) and higgsinolike LSP scenarios (right). The color code indicates the fraction of points

of points in each bin, so large fluctuations in the coverage are easily possible. The importance of $\tilde{t}_{1} \rightarrow t \tilde{\chi}_{i \neq 1}^{0}$ decays, followed by visible $\tilde{\chi}_{i \neq 1}^{0}$ decays, for SMODELS-allowed points is illustrated in the left plot in Fig. 10.

Turning to sbottoms, we see that the coverage is quite good for $m_{\tilde{b}_{1}} \lesssim 450 \mathrm{GeV}$ and $m_{\tilde{\chi}_{1}^{0}} \lesssim 250 \mathrm{GeV}$. For these mass ranges, $\tilde{b}_{1} \rightarrow b \tilde{\chi}_{1}^{0}$ (and/or $\tilde{b}_{1} \rightarrow t \tilde{\chi}_{1}^{-}$in the higgsinoLSP case) decays dominate. Once a larger variety of decay channels becomes relevant, the exclusion drops to about $50 \%$ of that of ATLAS. While results for $\tilde{b}_{1} \rightarrow t \tilde{\chi}_{1}^{-} \rightarrow t W \tilde{\chi}_{1}^{0}$ are available from ATLAS [41] and CMS [76], ${ }^{8}$ these are ULs for a same-sign lepton signature assuming both sbottoms decay via a chargino; they have a reach in sbottom mass of at most $500-550 \mathrm{GeV}$. It would be useful to have in addition simplified model results for $\tilde{b}_{1} \rightarrow b \tilde{\chi}_{i \neq 1}^{0} \rightarrow b Z \tilde{\chi}_{1}^{0}$ or $b h \tilde{\chi}_{1}^{0}$, best in the form of EMs for symmetric and asymmetric decay branches. The importance of these decay modes for SMODELS-allowed points is illustrated in the right plot in Fig. 10.

It is relevant to stress that the branching ratios shown in Fig. 10 only consider visible decays. In particular the higgsino-like LSP dataset contains many points where sbottom branching ratios are shared out in $\tilde{b} \rightarrow b \tilde{\chi}_{i \neq 1}^{0}$ and $t \tilde{\chi}^{-}$decays (contributing to the reduced coverage for $m_{\tilde{b}_{1}} \gtrsim$ $500 \mathrm{GeV}$ seen in Fig. 9) but the subsequent EW-ino decays are invisible because of mass compression. This leads to the patch of gray points just below the exclusion curve in the right plot of Fig. 10. Regardless of this, the conclusion from Fig. 10 is that EM results for stops and sbottoms decaying

\footnotetext{
8 We appreciate the fact that these are given for 3 different chargino masses.
}

Fraction of Higgsino LSP ATLAS excluded points excluded by SModelS

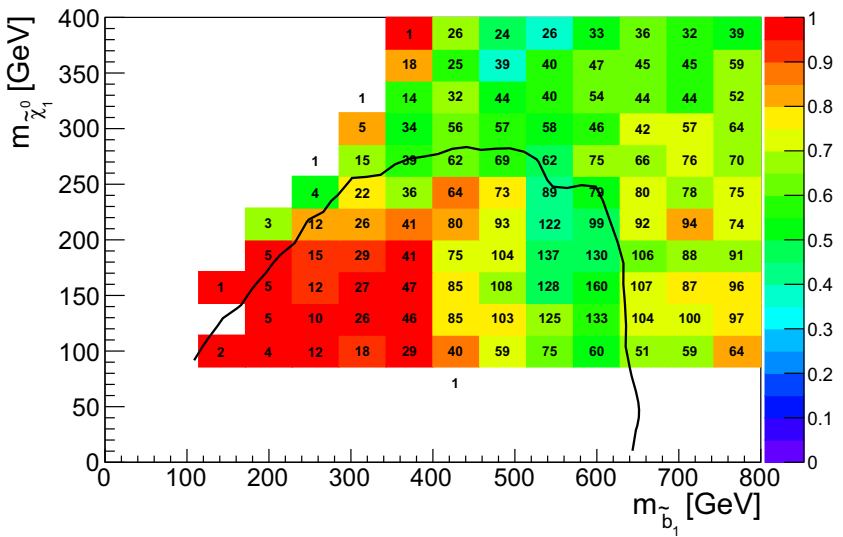

excluded by SMODELS as compared to ATLAS, while the text gives the total number of points tested in each bin. The black line is the 95\% CL exclusion line for the $\tilde{b}_{1} \rightarrow b \tilde{\chi}_{1}^{0}$ simplified model from [49]

through an intermediate particle (leading to final states with additional $W, Z$ or $h$ bosons) would be highly desirable.

\subsection{EW production}

It is also interesting to study how well EW production is covered by simplified models. To this end, we first show in Fig. 11 the coverage in the chargino vs. neutralino LSP mass plane. Here, the bino-like and higgsino-like LSP scenarios have been combined to increase the number of points. In the plot on the left, light charginos seem to be reasonably well constrained. However, this does not come from searches looking specifically for EW production, as is apparent from the plot on the right. The fact that the coverage does not follow the SMS exclusion curve is no surprise, as the latter was obtained for the best-case scenario of pure wino production. However, from the color code we see that the constraining power of EW searches is very poorly reproduced by SMS results. One of the reasons is that the SMS results typically assume strictly mass-degenerate $\tilde{\chi}_{1}^{ \pm}$and $\tilde{\chi}_{2}^{0}$, a condition which is rarely satisfied in the pMSSM. Moreover, $\operatorname{BR}\left(\tilde{\chi}_{i \neq 1}^{0} \rightarrow h \tilde{\chi}_{1}^{0}\right)$ is often sizable, which further reduces the coverage. (The SMS limit in the $W h+E_{T}^{\text {miss }}$ final state is effective only for very light LSP below $40 \mathrm{GeV}$ and cannot be combined with the limit on the $W Z+E_{T}^{\text {miss }}$ final state.) Finally, the 3 or 4 lepton searches in ATLAS do not have a jet veto; therefore in the ATLAS pMSSM study strong production may also feed into the EW exclusion, which is not the case in SMODELS for lack of the corresponding SMS results.

In Fig. 12 we show the same kind of plots for sleptons. Here, the coverage is quite good and reproduces reasonably well the SMS exclusion line for right sleptons. The exclusion line for left sleptons is naturally matched less well, because pMSSM points contain a mix of light left 


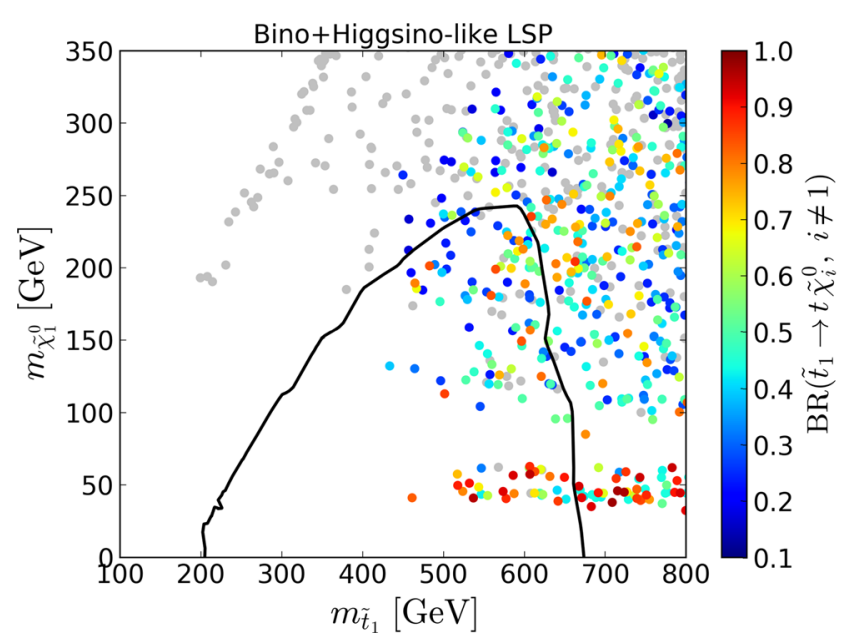

Fig. 10 Branching ratios of stop (left) and sbottom (right) decays into heavier neutralino mass eigenstates for SMODELS-allowed points, leading to signatures for which no SMS results are currently available $\left(m_{\tilde{\chi}_{i \neq 1}^{0}}-m_{\tilde{\chi}_{1}^{0}} \geq 5 \mathrm{GeV}\right)$. Here, bino- and higgsino-like LSP scenarios

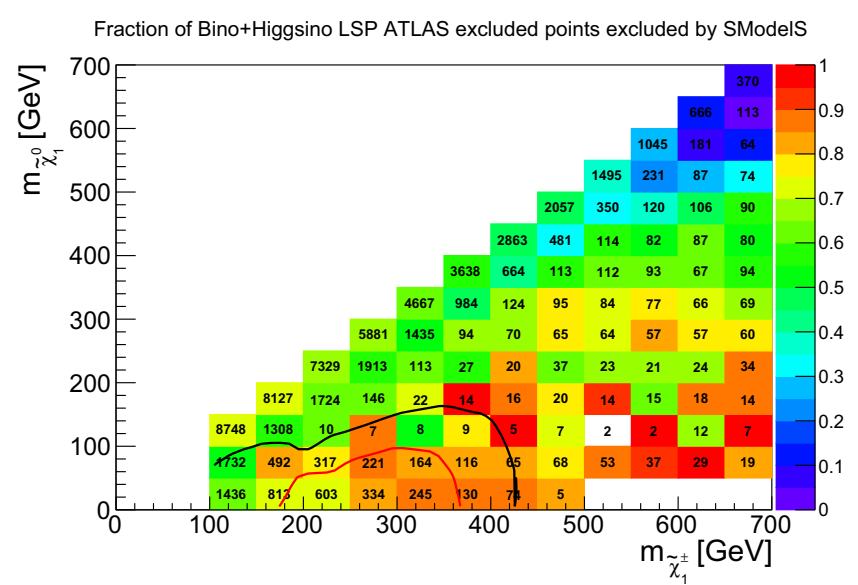

Fig. 11 Coverage in the chargino vs. neutralino mass plane, for $\tilde{\chi}_{1}^{ \pm}$ masses up to $700 \mathrm{GeV}$. Here, bino-like and higgsino-like LSP scenarios have been combined to increase the number of points. The plot on the left considers all analyses, the plot on the right only EW analyses. The color code indicates the fraction of points excluded by SMODELS

and right sleptons. Finally, a small fraction of points with $\min \left(m_{\tilde{l}}\right)=250-300 \mathrm{GeV}$ and light LSP escape exclusion in SMODELS because the sleptons partly undergo cascade decays via heavier EW-inos. Even if the direct decay into the LSP still dominates, the reduction in BR can be enough to result in $r<1$.

Last but not least we recall that EW cross sections are computed at leading order in SMODELS. Radiative corrections typically increase these cross sections by about $20 \%$, which slightly improves the coverage of the EW sector but does not change the overall picture. This is illustrated in Fig. 13, which shows the coverage of EW-inos and sleptons by EW analyses when rescaling the relevant $r$ values by $20 \%$.

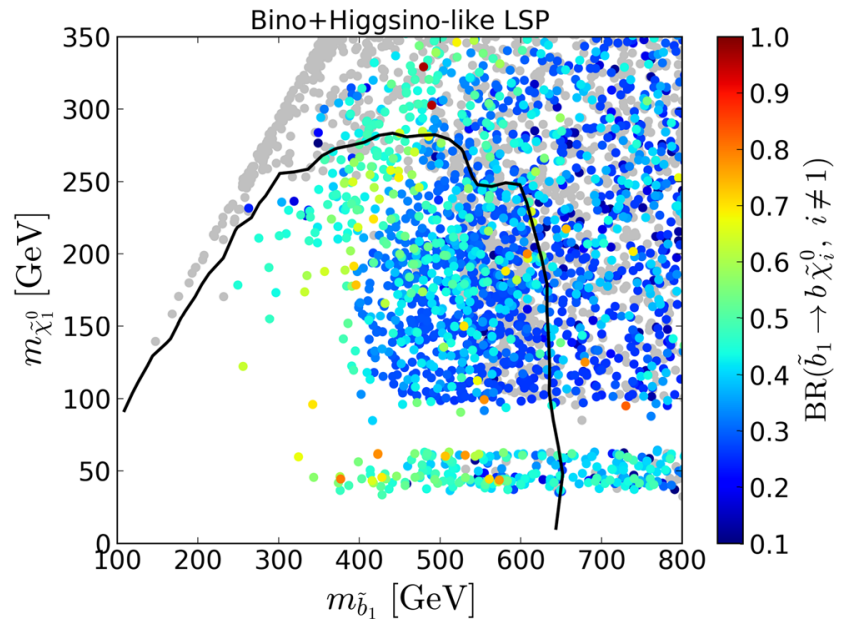

are combined. Gray points have BR $<10 \%$ for the decays considered. The black lines are the 95\% CL exclusion lines for the $\tilde{t}_{1} \rightarrow t \tilde{\chi}_{1}^{0}$ simplified model from [45] (left) and the $\tilde{b}_{1} \rightarrow b \tilde{\chi}_{1}^{0}$ simplified model from [49] (right). See text for details

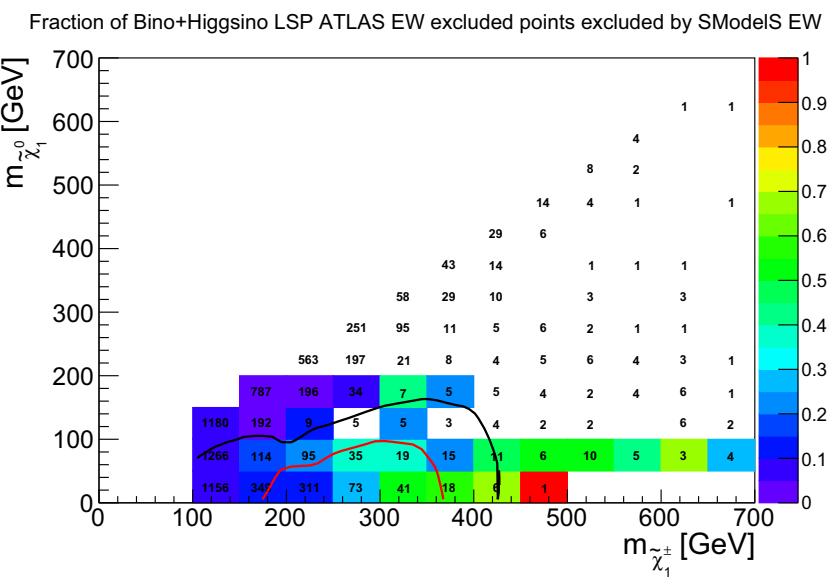

as compared to ATLAS, while the text gives the total number of points tested in each bin. For comparison, the exclusion line from the 3-leptons analysis [54] is shown in red and from the combination paper [84] is drawn in black

\section{Conclusions}

We studied to which extent the SUSY search results published by ATLAS and CMS in the context of SMS constraints actually cover the more realistic scenarios of a full model, concretely the phenomenological MSSM. To this end we analyzed the exclusion obtained with SMODELS $[9,10]$ with respect to the ATLAS pMSSM study [7]. From about $84 \mathrm{~K}$ pMSSM points excluded by ATLAS, the $8 \mathrm{TeV}$ results in SMODELS v1.1.1 exclude about 50K points. Efficiency maps proved to be important for constraining scenarios with a variety of production and/or decay modes, because they allow one to combine different contributions to the same sig- 


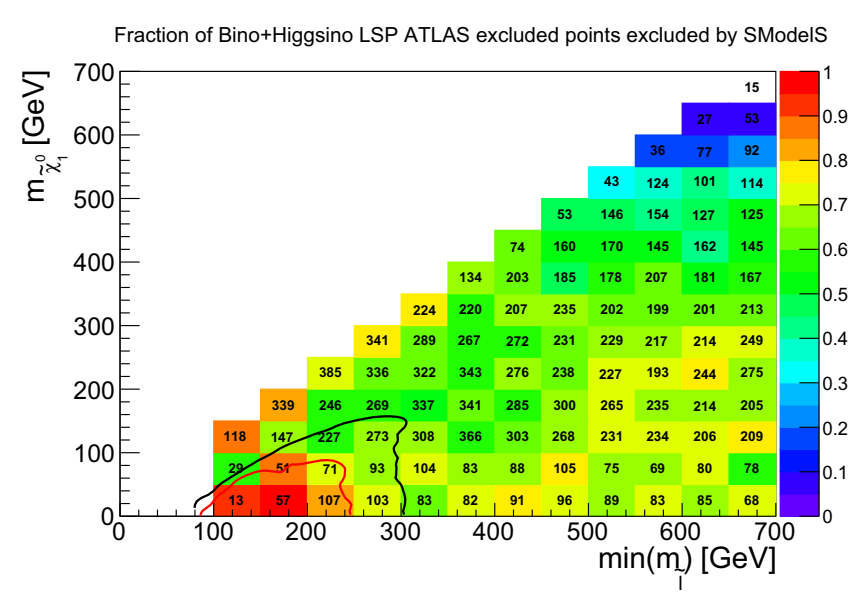

Fig. 12 Coverage in the plane of lightest slepton (first two generations) vs. LSP mass, for $\tilde{l}$ masses up to $700 \mathrm{GeV}$. Here, bino-like and higgsino-like LSP scenarios have been combined to increase the number of points. The plot on the left considers all analyses, the plot on the

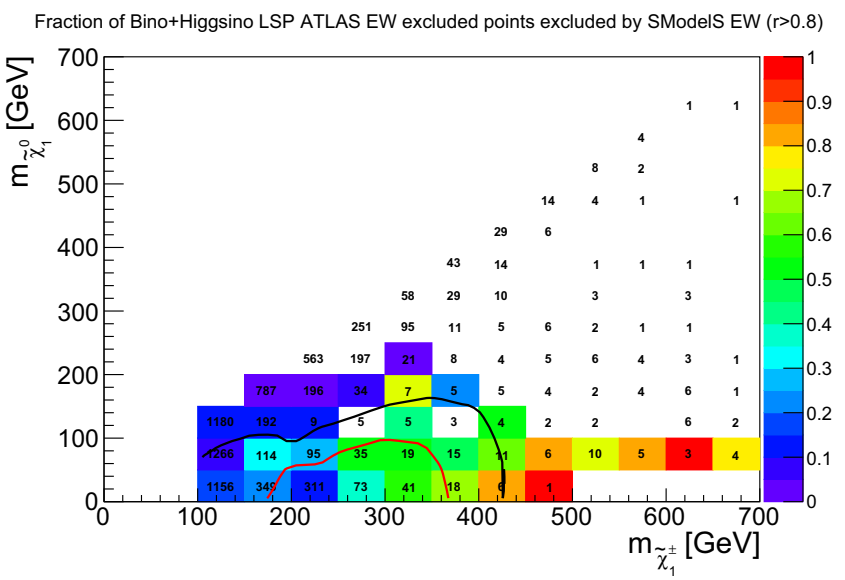

Fraction of Bino+Higgsino LSP ATLAS EW excluded points excluded by SModelS EW

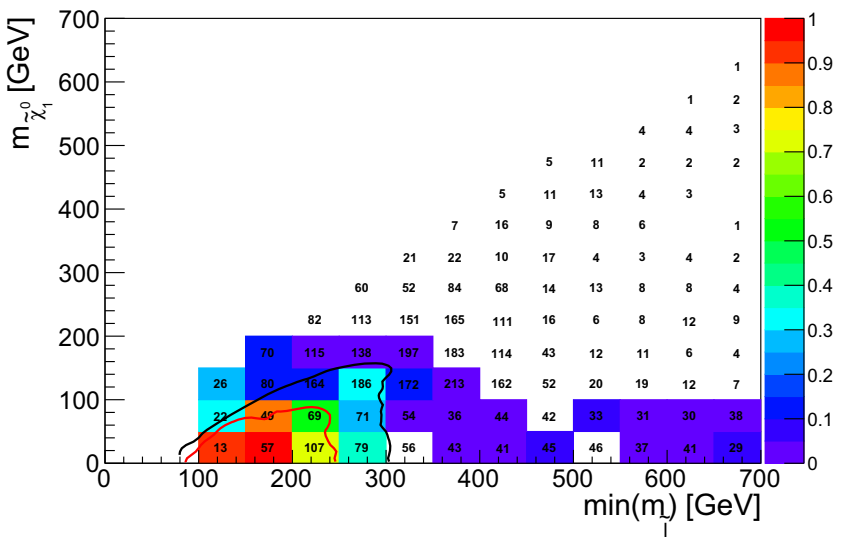

right only EW analyses. The color code indicates the fraction of points excluded by SMODELS as compared to ATLAS, while the text gives the total number of points tested in each bin. The exclusion lines for $\tilde{l}_{R}$ (red) and $\tilde{l}_{L}$ (black) are also shown for comparison

Fraction of Bino+Higgsino LSP ATLAS EW excluded points excluded by SModelS EW ( $r>0.8)$

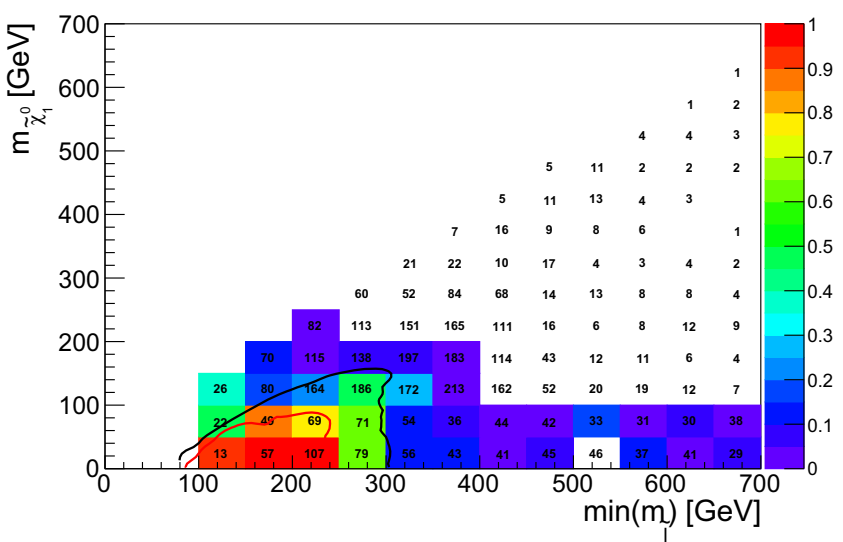

Fig. 13 Coverage of EW-inos and sleptons by EW analyses analogous to the right plots of Figs. 11 and 12 but considering points with $r_{\text {max }}>0.8$ (instead of $r_{\max }>1$ ) as excluded

nal region. Nonetheless, despite the plethora of SMS results available, about $40 \%$ of the points excluded by ATLAS are not excluded by SMODELS. These "escaping" points include gluinos as light as about $500 \mathrm{GeV}$, but also light stops/sbottoms or EW-inos with reasonably large cross sections. We analyzed the reasons for this limited coverage and how it might be improved.

Concretely, we found that a large part of the unconstrained cross section goes into simple but asymmetric topologies, either because pair-produced sparticles have two or more relevant decay modes, or because of associated production of two different sparticles. A particularly important case, for which no SMS results are currently available, is a 3-jet topology stemming from gluino-squark associated production with non-degenerate squarks: $p p \rightarrow \tilde{g} \tilde{q}$ followed by $\tilde{g} \rightarrow q \tilde{q} \rightarrow q \bar{q} \tilde{\chi}_{1}^{0}$ and $\tilde{q} \rightarrow q \tilde{\chi}_{1}^{0}$ when one of the squarks is lighter than the gluino, or $\tilde{g} \rightarrow g \tilde{\chi}_{1}^{0}$ and $\tilde{q} \rightarrow q \tilde{g} \rightarrow q g \tilde{\chi}_{1}^{0}$ otherwise. For one third of the bino-like LSP points which are excluded by ATLAS but not by SMODELS, this topology has a cross section $>20 \mathrm{fb}$.

For the case that the produced SUSY particles share out their branching ratios over several different decay modes, which need to be combined to obtain a good limit, we highlighted the example of stop and sbottom decays via heavier EW-inos, which in turn decay visibly into the LSP. While SMS results for stop-pair production with both stops decaying via an intermediate chargino exist, analogous results considering also $\tilde{t}_{1} \rightarrow t \tilde{\chi}_{2}^{0} \rightarrow t Z \tilde{\chi}_{1}^{0}, \tilde{b}_{1} \rightarrow b \tilde{\chi}_{2}^{0} \rightarrow b Z \tilde{\chi}_{1}^{0}$ or $\tilde{b}_{1} \rightarrow t \tilde{\chi}_{1}^{-} \rightarrow t W \tilde{\chi}_{1}^{0}$ decays are missing. Efficiency maps for these cases would be highly desirable to improve the coverage of the third generation.

Regarding the EW SUSY sector, the coverage of light sleptons by SMS results is quite good. For EW-inos, however, the situation is less satisfying. This might be improved if EMs 
were available for the EW-ino searches in multi-lepton channels instead of only UL-type results. Moreover, for multilepton searches without jet veto, EM results applicable also to $\mathrm{EW}$-inos stemming from strong production would be interesting.

The coverage in SMODELS may also be limited when the initially produced SUSY particles undergo a series of cascade decays leading to long decay chains with more than one intermediate sparticles. This situation is difficult to cover by simplified models, since it involves a large number of free parameters. Interestingly, we find that only a small fraction of the points which escape exclusion by SMODELS fall into this class. In this view it is much more useful to improve the constraining power of simple SMS (with few parameters) by providing, e.g., additional efficiency maps and sufficient mass-vs.-mass planes for a reliable interpolation in all mass dimensions, than to present results for more complicated topologies. Although complicated topologies (decay chains with more than three mass parameters) have been considered by the experimental collaborations, these results always assume very specific mass relations to limit the number of free parameters and hence cannot be used for generic scenarios.

Overall, the SMS approach provides a powerful means to quickly test the predictions of new physics models against the constraints from a large variety of experimental searches. However, not excluded by SMS results does not automatically mean allowed by all LHC searches; it is advisable to further test "surviving" points with Monte Carlo event simulation, if they have sizable cross sections. Implementations of ATLAS and CMS analyses in public recasting tools like CHECKMATE [85, 86], MADANALYSIS 5 [87,88], RIVET [89] (v2.5 onwards) and GAMBIT's ColliderBit [90,91] can be used to this end. Finally, these tools may also be used to produce additional SMS results beyond those provided by the experimental collaborations.

Acknowledgements We thank the ATLAS and CMS SUSY groups for providing a plethora of SMS cross section upper limits and efficiency maps in digital format. Moreover, we are particularly grateful for the detailed material on the ATLAS pMSSM study available on HepDATA, which made the analysis presented in this paper possible. This research was supported in part by the ANR project DMASTROLHC grant no. ANR-12-BS05-0006, the IN2P3 project "ThéorieLHCiTools" and the CNRS-FAPESP collaboration PRC275431. F.A. is supported by the Austrian FWF, project P26896-N27, Su.K. by the "New Frontiers" program of the Austrian Academy of Sciences, U.L. by the "Investissements d'avenir, Labex ENIGMASS", and A.L. by the São Paulo Research Foundation (FAPESP), projects 2015/20570-1 and 2016/50338-6.

Open Access This article is distributed under the terms of the Creative Commons Attribution 4.0 International License (http://creativecomm ons.org/licenses/by/4.0/), which permits unrestricted use, distribution, and reproduction in any medium, provided you give appropriate credit to the original author(s) and the source, provide a link to the Creative Commons license, and indicate if changes were made. Funded by SCOAP ${ }^{3}$.

\section{A Diagrams and processes for missing topologies}

Here we show the explicit diagrams, SUSY processes and labeling in SMODELS notation for the missing topologies of Fig. 7. 


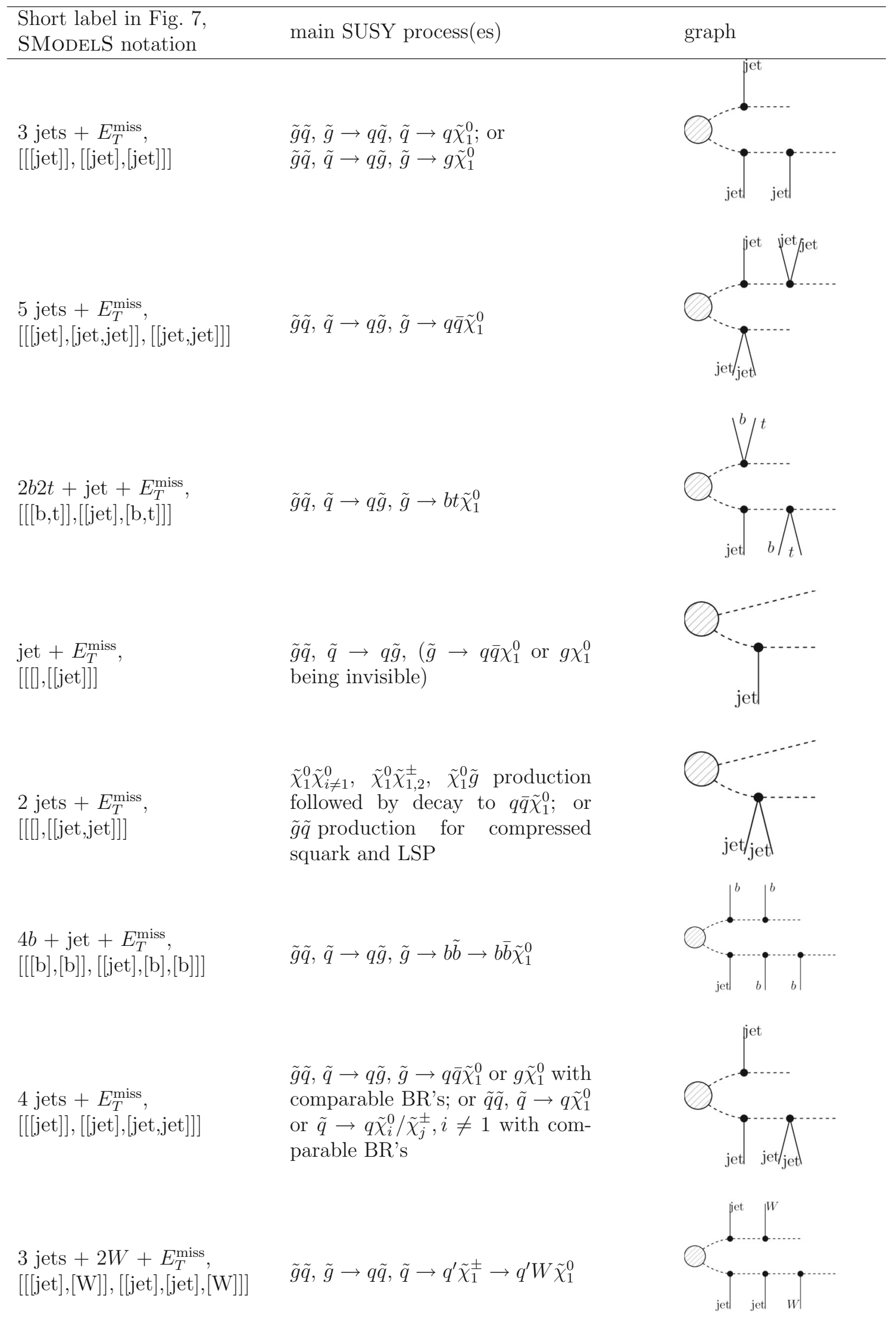


Table 5 Contributions of specific signal topologies to the total exclusion for the pMSSM point no. 192342466. The second column shows the topology cross section (production cross section times branching ratios), while the third column shows the topology contribution to the signal yield for the $2 j t$ signal region of Ref. [35]. The last column shows the $r$-values obtained using each topology individually. The last line shows the corresponding results including all possible gluino and squarks decays, resulting in a larger total cross section and $r$-value. See text for details

\begin{tabular}{llll}
\hline Topology & Cross section & Contribution to $2 j t(\%)$ & $r$-value \\
\hline$\tilde{g} \tilde{g} \rightarrow 2$ jets $+E_{T}^{\text {miss }}$ & $250 \mathrm{fb}$ & 21 & 0.37 \\
$\tilde{g} \tilde{q} \rightarrow 3$ jets $+E_{T}^{\text {miss }}$ & $664 \mathrm{fb}$ & 59 & 1.01 \\
$\tilde{q} \tilde{q} \rightarrow 4$ jets $+E_{T}^{\text {miss }}$ & $136 \mathrm{fb}$ & 4 & 0.08 \\
Total $(\tilde{g} \tilde{g}+\tilde{g} \tilde{q}+\tilde{q} \tilde{q})$ & $1220 \mathrm{fb}$ & 100 & 1.70 \\
\hline
\end{tabular}

\section{B Example for the impact of a 3 jets $+E_{T}^{\text {miss }}$ simplified model}

In order to illustrate the importance of asymmetric topologies, we analyze here in more detail one of the ATLASexcluded points with a light gluino which has not been excluded by the SMS results. The pMSSM point we consider is no. 192342466 of the bino-LSP dataset; it has light gluinos and a highly split spectrum of squarks with light $\tilde{q}_{L}$. Concretely,

$m_{\tilde{\chi}_{1}^{0}}=666, m_{\tilde{g}}=712, m_{\tilde{u}_{L}}=758, m_{\tilde{d}_{L}}=m_{\tilde{s}_{L}}=761$,

$m_{\tilde{d}_{R}}=1343, m_{\tilde{u}_{R}}=3968$,

where all values are in $\mathrm{GeV}$. Stops and sbottoms are heavy with $m_{\tilde{t}_{1}, \tilde{b}_{1}} \approx 1.4 \mathrm{TeV}$ and $m_{\tilde{t}_{2}, \tilde{b}_{2}} \approx 3.3 \mathrm{TeV}$. The wino- and higgsino-like EW-inos have masses around $3.5 \mathrm{TeV}$.

In the following we only consider production of gluinos and the squarks $\tilde{u}_{L}, \tilde{d}_{L}, \tilde{s}_{L}, \tilde{d}_{R}$, since this corresponds to $\simeq 95 \%$ of the total SUSY cross section for this point. For simplicity we will refer to the associated and pair production of these squarks as $\tilde{g} \tilde{q}$ and $\tilde{q} \tilde{q}$. The NLO+NLL cross section for gluino-pair production is $322 \mathrm{fb}$, while the $\tilde{g} \tilde{q}$ production cross section is $762 \mathrm{fb}$. The dominant gluino and squark decays are $\tilde{g} \rightarrow g+\tilde{\chi}_{1}^{0}(88 \% \mathrm{BR})$ and $\tilde{q} \rightarrow q \tilde{g}$ (99\% BR). As a result, a large fraction of the signal goes to the 3 jets + $E_{T}^{\text {miss }}$ final state discussed as missing topology in Sect. 3.1.

According to the ATLAS pMSSM study, this point is excluded by the 0-lepton $+2-6$ jets $+E_{T}^{\text {miss }}$ search [35] (ATLAS-SUSY-2013-02). This is also the analysis which gives the highest $r$ value in SMODELS, namely $r=0.36$ for the $\tilde{g} \tilde{g} \rightarrow 2$ jets $+E_{T}^{\text {miss }}$ topology. Hence this point is clearly not excluded by the SMS results. In order to investigate how specific topologies contribute to the total signal yield and to the exclusion of this point, we used the CHECKMATE 2 (v2.0.14) implementation of this analysis along with Pythia 8.230 and Delphes 3.4.1 for event generation and detector simulation. We generated signal events at leading order for associated and pair production of $\tilde{g}, \tilde{u}_{L}, \tilde{d}_{L}, \tilde{s}_{L}$ and $\tilde{d}_{R}$ and then rescaled the cross sections using the $\mathrm{K}$ factors computed with NLLFAST. For obtaining the $r$ values we used the numbers provided by CHECKMATE, including a $20 \%$ uncertainty for the theoretical cross sections, which corresponds to the value used in SMODELS when computing likelihoods.

In Table 5 we show, for this specific pMSSM point, the main contributions to the total signal yield for the best signal region (SR), $2 j t$, in ATLAS-SUSY-2013-02. As we can see, if we only consider the symmetric $\tilde{g} \tilde{g} \rightarrow 2$ jets + $E_{T}^{\text {miss }}$ topology, we obtain an $r$ value very similar to the one obtained by SMODELS $(r=0.37)$ and the point is far from being excluded. However, if we include the asymmetric $\tilde{g} \tilde{q} \rightarrow 3$ jets $+E_{T}^{\text {miss }}$ topology, the $r$ value increases to 1.38 and the point can be excluded. In contrast, the contribution from $\tilde{q} \tilde{q}$ production with $\tilde{q} \rightarrow q \tilde{g}, \tilde{g} \rightarrow g \tilde{\chi}_{1}^{0}$ has a tiny effect. For completeness, in the last line of Table 5 we also present the inclusive result, which incorporates all possible gluino and squark decays, thus giving a slightly higher $r$-value $(r=1.7)$.

In this example we can clearly see that the asymmetric $\tilde{g} \tilde{q}$ topology is the dominant one and essential for excluding the tested point. This illustrates how SMS results for $\tilde{g} \tilde{q}$ topologies, with unrelated gluino and squark masses, would help improve the coverage of the pMSSM. Particularly useful would be efficiency maps, as they allow one to combine different contributions to the same signal region in the simplified model context.

\section{References}

1. N. Arkani-Hamed, P. Schuster, N. Toro, J. Thaler, L.-T. Wang, B. Knuteson, S. Mrenna, MARMOSET: the path from LHC data to the new standard model via on-shell effective theories. arXiv:hep-ph/0703088

2. J. Alwall, P. Schuster, N. Toro, Simplified models for a first characterization of new physics at the LHC. Phys. Rev. D 79, 075020 (2009). arXiv:0810.3921

3. LHC New Physics Working Group Collaboration, D. Alves, Simplified models for LHC new physics searches. J. Phys. G 39, 105005 (2012). arXiv:1105.2838

4. ATLAS Collaboration, H. Okawa, Interpretations of SUSY searches in ATLAS with simplified models," in Particles and Fields. Proceedings, Meeting of the Division of the American Phys- 
ical Society, DPF 2011, Providence, USA, August 9-13, 2011. arXiv: 1110.0282

5. CMS Collaboration, S. Chatrchyan et al., Interpretation of searches for supersymmetry with simplified models. Phys. Rev. D 88(5), 052017 (2013). arXiv:1301.2175

6. MSSM Working Group Collaboration, A. Djouadi et al., The minimal supersymmetric standard model: group summary report, in GDR (Groupement De Recherche)—Supersymetrie, Montpellier, France, April 15-17, 1998. arXiv:hep-ph/9901246

7. ATLAS Collaboration, G. Aad et al., Summary of the ATLAS experiment's sensitivity to supersymmetry after LHC Run 1 interpreted in the phenomenological MSSM. JHEP 10, 134 (2015). arXiv: 1508.06608

8. http://hepdata.cedar.ac.uk/view/ins1389857. Accessed 13 Mar 2018

9. S. Kraml, S. Kulkarni, U. Laa, A. Lessa, W. Magerl, D. Proschofsky, W. Waltenberger, SModelS: a tool for interpreting simplifiedmodel results from the LHC and its application to supersymmetry. Eur. Phys. J. C 74, 2868 (2014). arXiv: 1312.4175

10. F. Ambrogi, S. Kraml, S. Kulkarni, U. Laa, A. Lessa, V. Magerl, J. Sonneveld, M. Traub, W. Waltenberger, SModelS v1.1 user manual. arXiv: 1701.06586

11. J. Heisig, A. Lessa, L. Quertenmont, Simplified models for exotic BSM searches. JHEP 12, 087 (2015). arXiv:1509.00473

12. G. Belanger, G. Drieu La Rochelle, B. Dumont, R.M. Godbole, S. Kraml, S. Kulkarni, LHC constraints on light neutralino dark matter in the MSSM. Phys. Lett. B 726, 773-780 (2013). arXiv:1308.3735

13. D. Barducci, G. Belanger, C. Hugonie, A. Pukhov, Status and prospects of the nMSSM after LHC Run-1. JHEP 01, 050 (2016). arXiv: 1510.00246

14. C. Arina, M.E.C. Catalan, S. Kraml, S. Kulkarni, U. Laa, Constraints on sneutrino dark matter from LHC Run 1. JHEP 05, 142 (2015). arXiv: 1503.02960

15. L. Edelhäuser, M. Krämer, J. Sonneveld, Simplified models for same-spin new physics scenarios. JHEP 04, 146 (2015). arXiv: 1501.03942

16. S. Kraml, U. Laa, L. Panizzi, H. Prager, Scalar versus fermionic top partner interpretations of $t \bar{t}+E_{T}$ searches at the LHC. JHEP 11, 107 (2016). arXiv: 1607.02050

17. L. Edelhäuser, J. Heisig, M. Krämer, L. Oymanns, J. Sonneveld, Constraining supersymmetry at the LHC with simplified models for squark production. JHEP 12, 022 (2014). arXiv:1410.0965

18. C.F. Berger, J.S. Gainer, J.L. Hewett, T.G. Rizzo, Supersymmetry without prejudice. JHEP 0902, 023 (2009). arXiv:0812.0980

19. M.W. Cahill-Rowley, J.L. Hewett, S. Hoeche, A. Ismail, T.G. Rizzo, The new look pMSSM with neutralino and gravitino LSPs. Eur. Phys. J. C 72, 2156 (2012). arXiv:1206.4321

20. M.W. Cahill-Rowley, J.L. Hewett, A. Ismail, T.G. Rizzo, More energy, more searches, but the phenomenological MSSM lives on. Phys. Rev. D 88(3), 035002 (2013). arXiv:1211.1981

21. M. Cahill-Rowley, J.L. Hewett, A. Ismail, T.G. Rizzo, Lessons and prospects from the pMSSM after LHC Run I. Phys. Rev. D 91(5), 055002 (2015). arXiv: 1407.4130

22. T. Sjostrand, S. Mrenna, P .Z. Skands, PYTHIA 6.4 Physics and Manual. JHEP 0605, 026 (2006). arXiv:hep-ph/0603175

23. T. Sjostrand, S. Ask, J.R. Christiansen, R. Corke, N. Desai, P. Ilten, S. Mrenna, S. Prestel, C.O. Rasmussen, P.Z. Skands, An introduction to PYTHIA 8.2. Comput. Phys. Commun 191, 159-177 (2015). arXiv: 1410.3012

24. http://pauli.uni-muenster.de/ akule_01/nllwiki/index.php/ NLL-fast. Accessed 13 Mar 2018

25. W. Beenakker, R. Hopker, M. Spira, P. Zerwas, Squark and gluino production at hadron colliders. Nucl. Phys. B 492, 51-103 (1997). arXiv:hep-ph/9610490
26. A. Kulesza, L. Motyka, Threshold resummation for squarkantisquark and gluino-pair production at the LHC. Phys. Rev. Lett 102, 111802 (2009). arXiv:0807.2405

27. A. Kulesza, L. Motyka, Soft gluon resummation for the production of gluino-gluino and squark-antisquark pairs at the LHC. Phys. Rev D80, 095004 (2009). arXiv:0905.4749

28. W. Beenakker, S. Brensing, M. Kramer, A. Kulesza, E. Laenen et al., Soft-gluon resummation for squark and gluino hadroproduction. JHEP 0912, 041 (2009). arXiv:0909.4418

29. W. Beenakker, S. Brensing, M. Kramer, A. Kulesza, E. Laenen et al., Squark and gluino hadroproduction. Int. J. Mod. Phys. A 26, 2637-2664 (2011). arXiv:1105.1110

30. W. Beenakker, M. Kramer, T. Plehn, M. Spira, P. Zerwas, Stop production at hadron colliders. Nucl. Phys. B 515, 3-14 (1998). arXiv:hep-ph/9710451

31. W. Beenakker, S. Brensing, M. Kramer, A. Kulesza, E. Laenen et al., Supersymmetric top and bottom squark production at hadron colliders. JHEP 1008, 098 (2010). arXiv:1006.4771

32. P.Z. Skands, B. Allanach, H. Baer, C. Balazs, G. Belanger et al., SUSY Les Houches accord: interfacing SUSY spectrum calculators, decay packages, and event generators. JHEP 0407, 036 (2004). arXiv:hep-ph/0311123

33. M. Papucci, K. Sakurai, A. Weiler, L. Zeune, Fastlim: a fast LHC limit calculator. Eur. Phys. J. C 74(11), 3163 (2014). arXiv: 1402.0492

34. http://smodels.hephy.at/wiki/ListOfAnalysesv111. Accessed 13 Mar 2018

35. ATLAS Collaboration, G. Aad et al., Search for squarks and gluinos with the ATLAS detector in final states with jets and missing transverse momentum using $\sqrt{s}=8 \mathrm{TeV}$ proton-proton collision data. JHEP 09, 176 (2014). arXiv:1405.7875

36. ATLAS Collaboration, G. Aad et al., Search for new phenomena in final states with large jet multiplicities and missing transverse momentum at $\sqrt{s}=8 \mathrm{TeV}$ proton-proton collisions using the ATLAS experiment. JHEP 10, 130 (2013). arXiv:1308.1841 [Erratum: JHEP 01, 109 (2014)]

37. ATLAS Collaboration, G. Aad et al., Search for squarks and gluinos in events with isolated leptons, jets and missing transverse momentum at $\sqrt{s}=8 \mathrm{TeV}$ with the ATLAS detector. JHEP 04, (2015) 116. arXiv: 1501.03555

38. ATLAS Collaboration, Search for strongly produced supersymmetric particles in decays with two leptons at $\sqrt{s}=8 \mathrm{TeV}$, Tech. Rep. ATLAS-CONF-2013-089. CERN, Geneva (2013)

39. ATLAS Collaboration, G. Aad et al., Search for supersymmetry in events with large missing transverse momentum, jets, and at least one tau lepton in $20 \mathrm{fb}^{-1}$ of $\sqrt{s}=8 \mathrm{TeV}$ proton-proton collision data with the ATLAS detector. JHEP 09, 103 (2014). arXiv: 1407.0603

40. ATLAS Collaboration, G. Aad et al., Search for supersymmetry at $\sqrt{s}=8 \mathrm{TeV}$ in final states with jets and two same-sign leptons or three leptons with the ATLAS detector. JHEP 06, 035 (2014). arXiv: 1404.2500

41. ATLAS Collaboration, Search for strongly produced superpartners in final states with two same sign leptons with the ATLAS detector using $21 \mathrm{fb}^{-1}$ of proton-proton collisions at $\sqrt{s}=8 \mathrm{TeV}$, Tech. Rep. ATLAS-CONF-2013-007. CERN, Geneva (2013)

42. ATLAS Collaboration, G. Aad et al., Search for strong production of supersymmetric particles in final states with missing transverse momentum and at least three $b$-jets at $\sqrt{s}=8 \mathrm{TeV}$ protonproton collisions with the ATLAS detector. JHEP 10, 024 (2014). arXiv: 1407.0600

43. ATLAS Collaboration, G. Aad et al., Search for new phenomena in final states with an energetic jet and large missing transverse momentum in $p p$ collisions at $\sqrt{s}=8 \mathrm{TeV}$ with the ATLAS detector. Eur. Phys. J. C 75(7), 299 (2015). arXiv:1502.01518 [Erratum: Eur. Phys. J. C 75(9), 408 (2015)] 
44. ATLAS Collaboration, G. Aad et al., Search for direct pair production of the top squark in all-hadronic final states in proton-proton collisions at $\sqrt{s}=8 \mathrm{TeV}$ with the ATLAS detector. JHEP 09, 015 (2014). arXiv:1406.1122

45. ATLAS Collaboration, G. Aad et al., Search for top squark pair production in final states with one isolated lepton, jets, and missing transverse momentum in $\sqrt{s}=8 \mathrm{TeV} p p$ collisions with the ATLAS detector. JHEP 11, 118 (2014). arXiv:1407.0583

46. ATLAS Collaboration, G. Aad et al., Search for direct top-squark pair production in final states with two leptons in $p p$ collisions at $\sqrt{s}=8 \mathrm{TeV}$ with the ATLAS detector. JHEP 06, 124 (2014). arXiv: 1403.4853

47. ATLAS Collaboration, G. Aad et al., Search for pair-produced third-generation squarks decaying via charm quarks or in compressed supersymmetric scenarios in $p p$ collisions at $\sqrt{s}=8$ TeV with the ATLAS detector. Phys. Rev. D 90(5), 052008 (2014). arXiv: 1407.0608

48. ATLAS Collaboration, G. Aad et al., Search for direct top squark pair production in events with a $Z$ boson, $b$-jets and missing transverse momentum in $\sqrt{s}=8 \mathrm{TeV} p p$ collisions with the ATLAS detector. Eur. Phys. J. C 74(6), 2883 (2014). arXiv:1403.5222

49. ATLAS Collaboration, G. Aad et al., Search for direct thirdgeneration squark pair production in final states with missing transverse momentum and two $b$-jets in $\sqrt{s}=8 \mathrm{TeV} p p$ collisions with the ATLAS detector. JHEP 10, 189 (2013). arXiv:1308.2631

50. ATLAS Collaboration, G. Aad et al., ATLAS Run 1 searches for direct pair production of third-generation squarks at the Large Hadron Collider. Eur. Phys. J. C 75(10), 510 (2015). arXiv:1506.08616 [Erratum: Eur. Phys. J. C 76(3), 153 (2016)]

51. ATLAS Collaboration, G. Aad et al., Search for direct pair production of a chargino and a neutralino decaying to the $125 \mathrm{GeV}$ Higgs boson in $\sqrt{s}=8 \mathrm{TeV} p p$ collisions with the ATLAS detector. Eur. Phys. J. C 75(5), 208 (2015). arXiv:1501.07110

52. ATLAS Collaboration, G. Aad et al., Search for direct production of charginos, neutralinos and sleptons in final states with two leptons and missing transverse momentum in $p p$ collisions at $\sqrt{s}=8 \mathrm{TeV}$ with the ATLAS detector. JHEP 05, 071 (2014). arxiv: 1403.5294

53. ATLAS Collaboration, G. Aad et al., "Search for the direct production of charginos, neutralinos and staus in final states with at least two hadronically decaying taus and missing transverse momentum in $p p$ collisions at $\sqrt{s}=8 \mathrm{TeV}$ with the ATLAS detector," JHEP10 096, (2014) arXiv:1407.0350

54. ATLAS Collaboration, G. Aad et al., Search for direct production of charginos and neutralinos in events with three leptons and missing transverse momentum in $\sqrt{s}=8 \mathrm{TeV} p p$ collisions with the ATLAS detector. JHEP 04, 169 (2014). arXiv:1402.7029

55. ATLAS Collaboration, G. Aad et al., Search for supersymmetry in events with four or more leptons in $\sqrt{s}=8 \mathrm{TeV} p p$ collisions with the ATLAS detector. Phys. Rev. D 90(5), 052001 (2014). arXiv: 1405.5086

56. ATLAS Collaboration, G. Aad et al., Search for charginos nearly mass degenerate with the lightest neutralino based on a disappearing-track signature in $p p$ collisions at $\sqrt{s}=8 \mathrm{TeV}$ with the ATLAS detector. Phys. Rev. D 88(11), 112006 (2013). arXiv: 1310.3675

57. ATLAS Collaboration, G. Aad et al., Searches for heavy longlived sleptons and R-Hadrons with the ATLAS detector in $p p$ collisions at $\sqrt{s}=7$ TeV. Phys. Lett. B 720, 277-308 (2013). arXiv: 1211.1597

58. ATLAS Collaboration, G. Aad et al., Searches for heavy longlived charged particles with the ATLAS detector in proton-proton collisions at $\sqrt{s}=8 \mathrm{TeV}$. JHEP 01, 068 (2015). arXiv:1411.6795

59. ATLAS Collaboration, G. Aad et al., Search for neutral Higgs bosons of the minimal supersymmetric standard model in $p p$ collisions at $\sqrt{s}=8 \mathrm{TeV}$ with the ATLAS detector. JHEP 11, 056 (2014). arXiv:1409.6064
60. ATLAS Collaboration, Search for squarks and gluinos with the ATLAS detector in final states with jets and missing transverse momentum and $20.3 \mathrm{fb}^{-1}$ of $\sqrt{s}=8 \mathrm{TeV}$ proton-proton collision data, Tech. Rep. ATLAS-CONF-2013-047. CERN, Geneva (2013)

61. ATLAS Collaboration, Search for new phenomena using final states with large jet multiplicities and missing transverse momentum with ATLAS in $20 \mathrm{fb}^{-1}$ of $\sqrt{s}=8 \mathrm{TeV}$ proton-proton collisions, Tech. Rep. ATLAS-CONF-2013-054. CERN, Geneva (2013)

62. ATLAS Collaboration, Search for squarks and gluinos in events with isolated leptons, jets and missing transverse momentum at $\sqrt{s}=8 \mathrm{TeV}$ with the ATLAS detector, Tech. Rep. ATLAS-CONF2013-062. CERN, Geneva (2013)

63. ATLAS Collaboration, Search for strong production of supersymmetric particles in final states with missing transverse momentum and at least three b-jets using $20.1 \mathrm{fb}^{-1}$ of $p p$ collisions at $\sqrt{s}=8$ $\mathrm{TeV}$ with the ATLAS Detector, Tech. Rep. ATLAS-CONF-2013061. CERN, Geneva (2013)

64. ATLAS Collaboration, Search for direct production of the top squark in the all-hadronic ttbar + etmiss final state in $21 \mathrm{fb}^{-1}$ of $p p$ collisions at $\sqrt{s}=8 \mathrm{TeV}$ with the ATLAS detector, Tech. Rep. ATLAS-CONF-2013-024. CERN, Geneva (2013)

65. ATLAS Collaboration, Search for direct top squark pair production in final states with one isolated lepton, jets, and missing transverse momentum in $\sqrt{s}=8 \mathrm{TeV} p p$ collisions using $21 \mathrm{fb}^{-1}$ of ATLAS data, Tech. Rep. ATLAS-CONF-2013-037. CERN, Geneva (2013)

66. ATLAS Collaboration, Search for direct top squark pair production in final states with two leptons in $\sqrt{s}=8 \mathrm{TeV} p p$ collisions using $20 \mathrm{fb}^{-1}$ of ATLAS data, Tech. Rep. ATLAS-CONF-2013048. CERN, Geneva (2013)

67. ATLAS Collaboration, Search for direct third generation squark pair production in final states with missing transverse momentum and two $b$-jets in $\sqrt{s}=8 \mathrm{TeV} p p$ collisions with the ATLAS detector, Tech. Rep. ATLAS-CONF-2013-053. CERN, Geneva (2013)

68. ATLAS Collaboration, Search for chargino and neutralino production in final states with one lepton, two b-jets consistent with a Higgs boson, and missing transverse momentum with the ATLAS detector in $20.3 \mathrm{fb}^{-1}$ of $\sqrt{s}=8 \mathrm{TeV} p p$ collisions, Tech. Rep. ATLAS-CONF-2013-093. CERN, Geneva (2013)

69. CMS Collaboration, S. Chatrchyan et al., Search for supersymmetry in hadronic final states with missing transverse energy using the variables $\alpha_{T}$ and $b$-quark multiplicity in $p p$ collisions at $\sqrt{s}=8$ TeV. Eur. Phys. J. C 73(9), 2568 (2013). arXiv:1303.2985

70. CMS Collaboration, S. Chatrchyan et al., Search for gluino mediated bottom- and top-squark production in multijet final states in $p p$ collisions at 8 TeV. Phys. Lett. B 725, 243-270 (2013). arXiv: 1305.2390

71. CMS Collaboration, S. Chatrchyan et al., Search for new physics in the multijet and missing transverse momentum final state in proton-proton collisions at $\sqrt{s}=8 \mathrm{TeV}$. JHEP 06, 055 (2014). arXiv: 1402.4770

72. CMS Collaboration, V. Khachatryan et al., Searches for supersymmetry using the $M_{T 2}$ variable in hadronic events produced in $p p$ collisions at 8 TeV. JHEP 05, 078 (2015). arXiv: 1502.04358

73. CMS Collaboration, V. Khachatryan et al., Search for supersymmetry using razor variables in events with $b$-tagged jets in $p p$ collisions at $\sqrt{s}=8$ TeV. Phys. Rev. D 91, 052018 (2015). arXiv: 1502.00300

74. CMS Collaboration, Search for supersymmetry in $p p$ collisions at $8 \mathrm{TeV}$ in events with a single lepton, multiple jets and b-tags, Tech. Rep. CMS-PAS-SUS-13-007. CERN, Geneva (2013)

75. CMS Collaboration, Search for supersymmetry in pp collisions at $\sqrt{s}=8 \mathrm{TeV}$ in events with two opposite sign leptons, large number of jets, $b$-tagged jets, and large missing transverse energy, Tech. Rep. CMS-PAS-SUS-13-016. CERN, Geneva (2013)

76. CMS Collaboration, S. Chatrchyan et al., Search for new physics in events with same-sign dileptons and jets in pp collisions at $\sqrt{s}=8$ 
TeV. JHEP 01, 163 (2014). arXiv:1311.6736 [Erratum: JHEP 01, 014 (2015)]

77. CMS Collaboration, V. Khachatryan et al., Searches for supersymmetry based on events with $b$-jets and four $W$ bosons in $p p$ collisions at 8 TeV. Phys. Lett. B 745, 5-28 (2015). arXiv:1412.4109

78. CMS Collaboration, Search for top squarks in multijet events with large missing momentum in proton-proton collisions at $8 \mathrm{TeV}$, Tech. Rep. CMS-PAS-SUS-13-015. CERN, Geneva (2013)

79. CMS Collaboration, A search for scalar top quark production and decay to all hadronic final states in $p p$ collisions at $\sqrt{s}=8 \mathrm{TeV}$, Tech. Rep. CMS-PAS-SUS-13-023. CERN, Geneva (2015)

80. CMS Collaboration, S. Chatrchyan et al., Search for top-squark pair production in the single-lepton final state in pp collisions at $\sqrt{s}=8$ TeV. Eur. Phys. J. C 73(12), 2677 (2013). arXiv:1308.1586

81. CMS Collaboration, Search for direct production of bottom squark pairs, Tech. Rep. CMS-PAS-SUS-13-018. CERN, Geneva (2014)

82. CMS Collaboration, V. Khachatryan et al., Search for supersymmetry in events with soft leptons, low jet multiplicity, and missing transverse energy in proton-proton collisions at $\sqrt{s}=8 \mathrm{TeV}$. Phys. Lett. B 759, 9-35 (2016). arXiv:1512.08002

83. CMS Collaboration, V. Khachatryan et al., Searches for electroweak production of charginos, neutralinos, and sleptons decaying to leptons and $W, Z$, and Higgs bosons in $p p$ collisions at 8 TeV. Eur. Phys. J. C 74(9), 3036 (2014). arXiv:1405.7570

84. ATLAS Collaboration, G. Aad et al., Search for the electroweak production of supersymmetric particles in $\sqrt{s}=8 \mathrm{TeV} p p$ collisions with the ATLAS detector. Phys. Rev. D 93(5), 052002 (2016). arXiv: 1509.07152
85. M. Drees, H. Dreiner, D. Schmeier, J. Tattersall, J.S. Kim, CheckMATE: confronting your favourite new physics model with LHC data. Comput. Phys. Commun. 187, 227-265 (2015). arXiv: 1312.2591

86. D. Dercks, N. Desai, J.S. Kim, K. Rolbiecki, J. Tattersall, T. Weber, CheckMATE 2: from the model to the limit. arXiv:1611.09856

87. E. Conte, B. Dumont, B. Fuks, C. Wymant, Designing and recasting LHC analyses with MadAnalysis 5. Eur. Phys. J. C 74(10), 3103 (2014). arXiv: 1405.3982

88. B. Dumont, B. Fuks, S. Kraml, S. Bein, G. Chalons, E. Conte, S. Kulkarni, D. Sengupta, C. Wymant, Toward a public analysis database for LHC new physics searches using MADANALYSIS 5. Eur. Phys. J. C 75(2), 56 (2015). arXiv: 1407.3278

89. A. Buckley, J. Butterworth, L. Lonnblad, D. Grellscheid, H. Hoeth, J. Monk, H. Schulz, F. Siegert, Rivet user manual. Comput. Phys. Commun. 184, 2803-2819 (2013). arXiv:1003.0694

90. GAMBIT Collaboration, P. Athron et al., GAMBIT: the global and modular beyond-the-standard-model inference tool. arXiv: 1705.07908

91. C. Balazs et al., ColliderBit: a GAMBIT module for the calculation of high-energy collider observables and likelihoods. arXiv:1705.07919 